Article

\title{
Study on the Microwave-Assisted Batch and Continuous Flow Synthesis of $N$-Alkyl-Isoindolin-1-One-3-Phosphonates by a Special Kabachnik-Fields Condensation
}

\author{
Ádám Tajti ${ }^{1}{ }^{1}$, Nóra Tóth ${ }^{1}$, Bettina Rávai ${ }^{1}$, István Csontos ${ }^{1}$, Pál Tamás Szabó ${ }^{2}$ and \\ Erika Bálint 1,*(D) \\ 1 Department of Organic Chemistry and Technology, Budapest University of Technology and Economics, \\ 1521 Budapest, Hungary; tajti.adam@mail.bme.hu (Á.T.); toth.nora@mail.bme.hu (N.T.); \\ betti.ravai08@gmail.com (B.R.); icsontos@mail.bme.hu (I.C.) \\ 2 MS Metabolomics Laboratory, Instrumentation Center, Research Centre for Natural Sciences, Hungarian \\ Academy of Sciences, Magyar tudósok krt. 2., H-1117 Budapest, Hungary; szabo.pal@ttk.mta.hu \\ * Correspondence: ebalint@mail.bme.hu; Tel.: +36-1-463-3653
}

Academic Editor: Derek J. McPhee

Received: 30 June 2020; Accepted: 17 July 2020; Published: 21 July 2020

\begin{abstract}
A simple and efficient microwave (MW)-assisted method was elaborated for the catalyst-free synthesis of isoindolin-1-one-3-phosphonates by the three-component condensation of 2 -formylbenzoic acid, aliphatic primary amines and various dialkyl phosphites. The batch and the continuous flow reactions were optimized in respect of the temperature, the reaction time and the molar ratio of the starting materials. To evaluate the potential of MW irradiation, comparative thermal experiments were also carried out. In order to obtain "real time" information about the condensation, the special Kabachnik-Fields reaction of 2-formylbenzoic acid, butylamine and diethyl phosphite was monitored by in situ FT-IR spectroscopy. The novel title compounds could be prepared in high yields at low temperature under a short reaction time. A suitable method could also be developed for the preparation of the isoindolin-1-one-3-phosphonates at a "few $\mathrm{g}$ " scale by using a continuous flow MW reactor.
\end{abstract}

Keywords: isoindolin-1-one phosphonates; $\alpha$-aminophosphonate derivatives; three-component condensation; Kabachnik-Fields reaction; microwave; in situ FT-IR spectroscopy; continuous flow microwave reactor

\section{Introduction}

Isoindolin-1-ones (1) and their derivatives, such as 3-acyl- and 3-alkylidene-isoindolin-1-ones, may be considered as an important compound family among $N$-heterocycles [1-4] (Figure 1). This moiety can be found in a number of biologically active natural compounds and synthetic derivatives as well [5-7]. As a special subgroup, isoindolin-1-ones bearing a carboxylic ester or carboxylic amide function (2) may show anticancer- [5], antiarrhythmic- [8] or sodium channel modulator activities [9]. It is known that phosphorus analogues of biologically active carboxylic acid derivatives may often show important effects, e.g., $\alpha$-aminophosphonates and $\alpha$-amino acids [10]. Isoindolin-1-one phosphonates (3) - the P-analogues of compound 2-have been tried out as plant growth regulators [11]. 


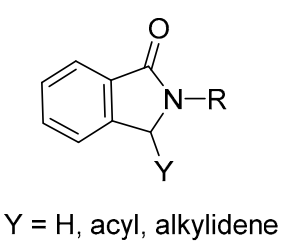

1<smiles>[Y]C(=O)C1c2ccccc2C(=O)N1[R]</smiles>

2<smiles>[R]N1C(=O)c2ccccc2C1[PH]([R])([R])[O-]</smiles>

3

Figure 1. General formula of isoindolin-1-one derivatives (1), isoindolin-1-one carboxylates (2) and isoindolin-1-one phosphonates (3).

Only a few examples have been reported on the synthesis of isoindolin-1-one phosphonates by the three-component condensation of 2-formylbenzoic acid, amines and dialkyl phosphites (Scheme 1/A) [7,12-17]. This process is somewhat analogous to the 'regular' Kabachnik-Fields reaction [18]; however, the carboxyl group of the 2-formylbenzoic acid allows a special ring closure step, as the speciality of this transformation. Ordóñez and co-workers carried out the condensation of 2-formylbenzoic acid, dimethyl phosphite and aromatic amines or aminoacetaldehyde dimethyl acetal under solvent-free conditions or in methanol in a microwave (MW) reactor [12,13]. The special Kabachnik-Fields reaction starting from amino alcohols [14] or chiral amines was also investigated using dimethyl phosphite as the phosphorus reagent [15]. In a few cases, the transformations were investigated in the presence of $\mathrm{NaH}$ [16], $\mathrm{T}_{3} \mathrm{P}^{\circledR}$ [17] or OSU-6 [7] as catalysts or additives. To the best of our knowledge, the three-component reaction of 2-formylbenzoic acid, aliphatic amines (butyl-, cyclohexyl- or benzylamine) and dialkyl phosphites, such as diisopropyl-, dibutyl- or dibenzyl phosphite, has not been studied comprehensively before.
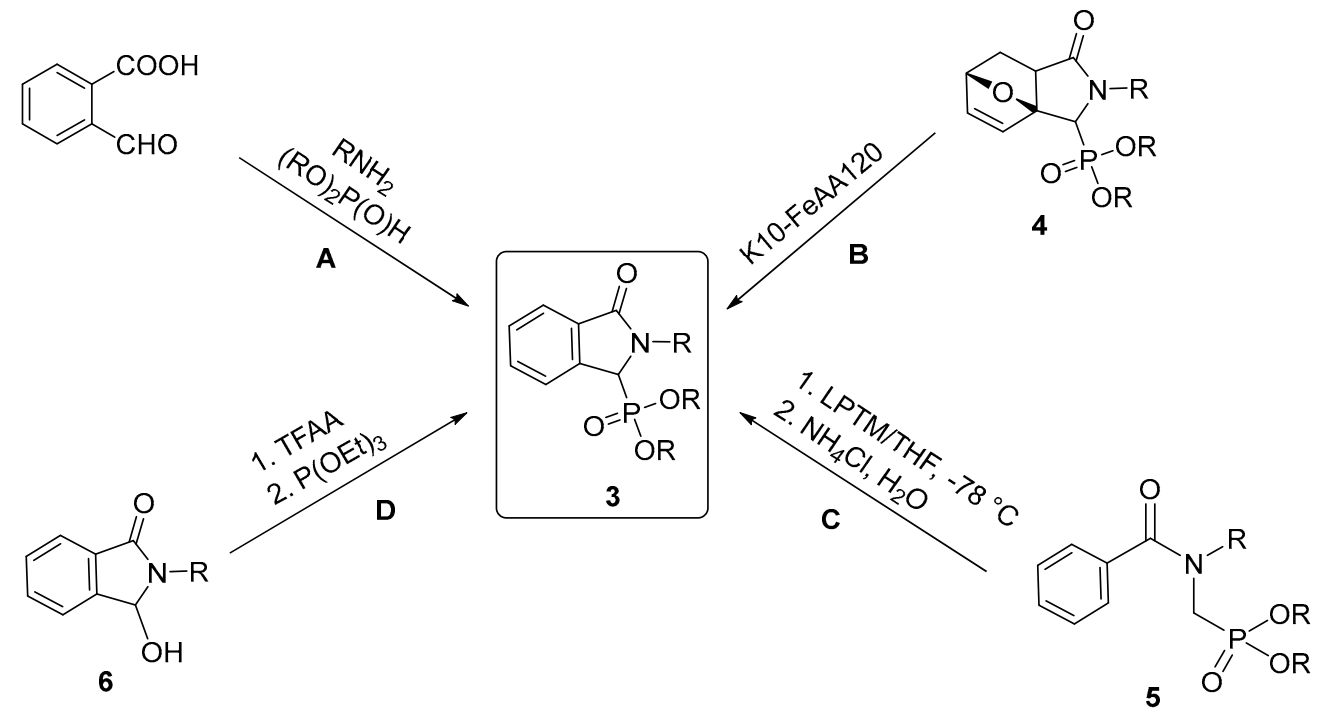

Scheme 1. Synthetic routes of isoindolin-1-one phosphonates (3).

Beside the three-component condensation, a few other synthetic routes are also known for the preparation of isoindolin-1-one phosphonates (3), such as the ring opening of tricyclic oxanorbornenes (4) (Scheme 1/B) [19], the ring closure of $\alpha$-amidophosphonates (5) (Scheme 1/C) [20] or the reaction of trialkyl phosphites with 3-hydroxy-isoindolin-1-ones (6) (Scheme 1/D) [21,22]. In these examples, the target compounds (3) were prepared starting from complex molecules (4-6), which were usually obtained after many reaction steps.

The application of the MW technique may be advantageous in several organic reactions and organophosphorus transformations as well [23]. The reactions may proceed faster and the use of a catalyst and/or a solvent is often evitable [24]. In case of multicomponent reactions, the MW irradiation may also be useful, as the corresponding products can usually be obtained more selectively. 
Therefore, the development of MW-assisted variations of multicomponent reactions is of growing interest [25]. The scale-up of MW-assisted transformations is a real challenge due to the limited geometry of the MW unit [26]. This problem can be solved by the use of continuous flow MW reactors [27]. In the last decade, various applications of the continuous flow MW technique were reported; however, in most cases non-professional MW reactors were applied, in which the parameters cannot be measured accurately [28]. In our previous work, we introduced a MW-assisted continuous flow synthesis of simple $\alpha$-aryl- $\alpha$-aminophosphonates by the three-component Kabachnik-Fields reaction [29], which may mean a good starting point towards the development of special condensation-ring closure reactions in continuous flow.

In this paper, at first, the catalyst- and solvent-free condensation of 2-formylbenzoic acid, aliphatic primary amines and various $>\mathrm{P}(\mathrm{O}) \mathrm{H}$ reagents was studied in a batch $\mathrm{MW}$ reactor. Our aim was to optimize the process and to synthesize and characterize new derivatives. The effect of the MW irradiation was also investigated; thus, comparative thermal experiments were also carried out. We also aimed at the monitoring of the special Kabachnik-Fields reaction by in situ FT-IR spectroscopy. Furthermore, we wished to develop a continuous flow MW-assisted method for the three-component reaction of 2-formylbenzoic acid, aliphatic amines and diethyl phosphite to obtain the corresponding isoindolin-1-one-3-phosphonates at a higher productivity.

\section{Results and Discussion}

\subsection{Synthesis of Isoindolin-1-one-3-phosphonates}

As the initial step of our work, the special Kabachnik-Fields reaction of 2-formylbenzoic acid, aliphatic primary amines, such as butyl-, cyclohexyl- or benzylamine, and diethyl phosphite was studied under catalyst- and solvent-free conditions (Table 1). The condensations were optimized in respect of the molar ratio of the starting materials, the temperature and the reaction time.

Table 1. Optimization of the condensation of 2-formylbenzoic acid, aliphatic primary amines and diethyl phosphite in a batch microwave reactor.

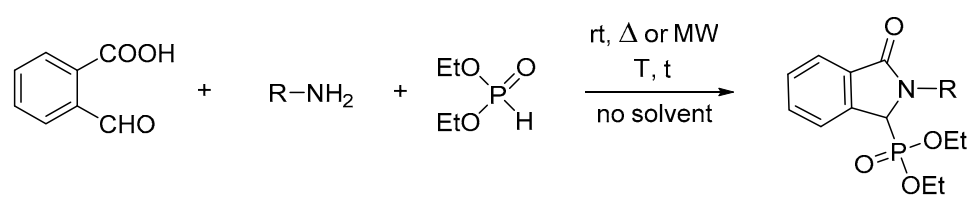

\begin{tabular}{ccccccccc}
\hline Entry & $\begin{array}{c}\text { Mode of } \\
\text { Heating }\end{array}$ & $\mathbf{R}$ & $\begin{array}{c}\text { Amine } \\
\text { (equiv) }\end{array}$ & $\begin{array}{c}\text { DEP } \\
\text { (equiv) }\end{array}$ & $\begin{array}{c}\mathbf{T} \\
\left({ }^{\circ} \mathbf{C}\right)\end{array}$ & $\mathbf{t}$ (min) & $\begin{array}{c}\text { Conversion }{ }^{\mathbf{a}} \\
\mathbf{( \% )}\end{array}$ & $\begin{array}{c}\text { Yield }^{\mathbf{b}} \\
\mathbf{( \% )}\end{array}$ \\
\hline 1 & - & $\mathrm{Bu}$ & 1 & 1 & 25 & $180^{c}$ & 77 & - \\
2 & $\mathrm{MW}$ & $\mathrm{Bu}$ & 1 & 1 & 40 & 10 & 61 & - \\
3 & $\mathrm{MW}$ & $\mathrm{Bu}$ & 1 & 1 & 60 & 10 & 85 & - \\
4 & $\mathrm{MW}$ & $\mathrm{Bu}$ & 1 & 1 & 80 & 10 & 87 & - \\
5 & $\mathrm{MW}$ & $\mathrm{Bu}$ & 1 & 1 & 60 & 20 & 90 & - \\
6 & $\mathrm{MW}$ & $\mathrm{Bu}$ & 1 & 1 & 60 & 30 & 91 & - \\
7 & $\mathrm{MW}$ & $\mathrm{Bu}$ & 1 & 1.1 & 60 & 10 & 87 & - \\
8 & $\mathrm{MW}$ & $\mathrm{Bu}$ & 1 & 1.2 & 60 & 10 & 89 & - \\
9 & $\mathrm{MW}$ & $\mathrm{Bu}$ & 1.1 & 1 & 60 & 10 & 92 & - \\
10 & $\mathrm{MW}$ & $\mathrm{Bu}$ & 1.2 & 1 & 60 & 10 & 100 & $94(\mathbf{7 a})$ \\
11 & - & $\mathrm{Bu}$ & 1.2 & 1 & 60 & 10 & 90 & - \\
12 & $\mathrm{MW}$ & ${ }^{c} \mathrm{Hex}$ & 1.2 & 1 & 60 & 10 & 82 & - \\
13 & $\mathrm{MW}$ & ${ }^{c} \mathrm{Hex}$ & 1.2 & 1 & 60 & 20 & 93 & - \\
14 & $\mathrm{MW}$ & ${ }^{c} \mathrm{Hex}$ & 1.2 & 1 & 60 & 30 & 100 & $84(\mathbf{8 a})$ \\
15 & - & ${ }^{c} \mathrm{Hex}$ & 1.2 & 1 & 60 & 30 & 84 & - \\
16 & $\mathrm{MW}$ & $\mathrm{Bn}$ & 1.2 & 1 & 60 & 10 & 89 & - \\
17 & $\mathrm{MW}$ & $\mathrm{Bn}$ & 1.2 & 1 & 60 & 20 & 100 & $90(\mathbf{9 a})$ \\
18 & - & $\mathrm{Bn}$ & 1.2 & 1 & 60 & 20 & 88 & - \\
\hline
\end{tabular}

${ }^{\mathrm{a}}$ On the basis of GC; ${ }^{\mathrm{b}}$ After column chromatography; ${ }^{\mathrm{c}}$ No change after longer reaction time. 
At first, the 2-formylbenzoic acid was reacted with butylamine and diethyl phosphite in a molar ratio of 1:1:1 at room temperature for $3 \mathrm{~h}$, and the conversion was only $77 \%$ (Table 1 , Entry 1). Carrying out the reaction at a higher temperature of $40{ }^{\circ} \mathrm{C}$ under $\mathrm{MW}$ irradiation, $61 \%$ of diethyl (2-butyl-3-oxo-2,3-dihydro-1H-isoindol-1-yl)phosphonate (7a) was obtained after $10 \mathrm{~min}$ (Table 1, Entry 2). Repeating the condensation at $60{ }^{\circ} \mathrm{C}$ for $10 \mathrm{~min}$, the conversion increased to $85 \%$ (Table 1, Entry 3). Applying a higher temperature of $80{ }^{\circ} \mathrm{C}$ for the same reaction time, the conversion did not change significantly (Table 1, Entry 4). Performing the condensation at $60^{\circ} \mathrm{C}$ using longer reaction times (20 or $30 \mathrm{~min}$ ), the proportion of the isoindolin-1-one-3-phosphonate (7a) was somewhat higher (90\% and 91\%, respectively) as compared to the reaction carried out for $10 \mathrm{~min}$ (Table 1, Entries 5 and 6 vs. Entry 3). In the next experiments, the effect of the molar ratio of the starting materials was studied at $60{ }^{\circ} \mathrm{C}$ applying a reaction time of 10 min (Table 1 , Entries 7-10). Increasing the amount of diethyl phosphite to 1.1 or 1.2 equivalents had no effect on the conversion (Table 1, Entry 3 vs. Entries 7 and 8). However, by using a small excess of butylamine (1.1 equivalents), an increase in the conversion was observed, and applying 1.2 equivalents of the amine component, a complete conversion could be achieved (Table 1, Entries 9 and 10). The diethyl (2-butyl-3-oxo-2,3-dihydro-1H-isoindol-1-yl)phosphonate (7a) was isolated in a yield of 94\% (Table 1, Entry 10). To evaluate the potential of the MW irradiation, the same reaction was repeated using conventional heating, resulting in a conversion of only $90 \%$ (Table 1, Entry 11).

Carrying out the condensation of the 2-formylbenzoic with 1.2 equivalents of cyclohexylamine and 1 equivalent of diethyl phosphite at $60{ }^{\circ} \mathrm{C}$ for $10 \mathrm{~min}, 82 \%$ of diethyl (2-cyclohexyl-3-oxo-2,3-dihydro-1H-isoindol-1-yl)phosphonate (8a) was formed (Table 1, Entry 12). After an irradiation of $20 \mathrm{~min}, 93 \%$ of $\mathbf{8 a}$ was present in the reaction mixture (Table 1, Entry 13), while after $30 \mathrm{~min}$, a complete conversion was achieved (Table 1, Entry 14). The corresponding isoindolin-1-one-3-phosphonate (8a) could be obtained in a yield of $84 \%$ after column chromatography. The comparative thermal experiment reached a conversion of only $84 \%$ (Table 1, Entry 15).

Applying benzylamine, a reaction time of $10 \mathrm{~min}$ was also not enough for a complete conversion; however, after $20 \mathrm{~min}$ the proportion of the diethyl (2-benzyl-3-oxo-2,3-dihydro$1 H$-isoindol-1-yl)phosphonate (9a) was 100\% (Table 1, Entries 16 and 17). Product 9a was isolated in a yield of $90 \%$. The thermal reaction was somewhat slower, the conversion was only $88 \%$ after 20 min (Table 1, Entry 18).

Based on the results obtained, all the three aliphatic amines allowed fast transformation towards the desired isoindolin-1-one phosphonates (7a-9a). The butylamine was the most reactive amine, as the special Kabachnik-Fields reaction was already complete after $10 \mathrm{~min}$, and the cyclohexylamine was the less reactive, possibly due to the steric hindrance of the bulky cyclohexyl group. The conversions in the comparative thermal experiments were by $10-16 \%$ lower, which clearly indicates the efficiency of the MW technique.

In the next series of experiments, the three-component condensation of 2-formylbenzoic acid, primary amines and various dialkyl phosphites was studied using the optimized conditions (60 $\left.{ }^{\circ} \mathrm{C}, 10-30 \mathrm{~min}\right)$ (Scheme 2). The reactions were complete in all cases. Carrying out the condensation of 2-formylbenzoic acid and butylamine with dimethyl phosphite, the dimethyl (2-butyl-3-oxo-2,3-dihydro-1H-isoindol-1-yl)phosphonate (7b) was obtained in a yield of $85 \%$ after column chromatography. Changing for diisopropyl- or dibutyl phosphite, the corresponding isoindolin-1-one-3-phosphonates (7c and 7d) were synthesized in yields of $89 \%$ and 91\%, respectively. Dibenzyl phosphite was also tried out as the P-reagent. In this case, the corresponding isoindolin-1-one phosphonate (7d) could be prepared in a slightly lower yield (81\%). Performing the experiments starting from cyclohexylamine, the condensations were slower (30 min), and the desired isoindolin-1-one-3-phosphonates $(\mathbf{8 b}-\mathbf{e})$ were isolated in yields of $71-80 \%$. The condensation was also extended for benzylamine, obtaining isoindolin-1-one phosphonates $\mathbf{9 b}-\mathbf{e}$ in high yields (80-85\%). 


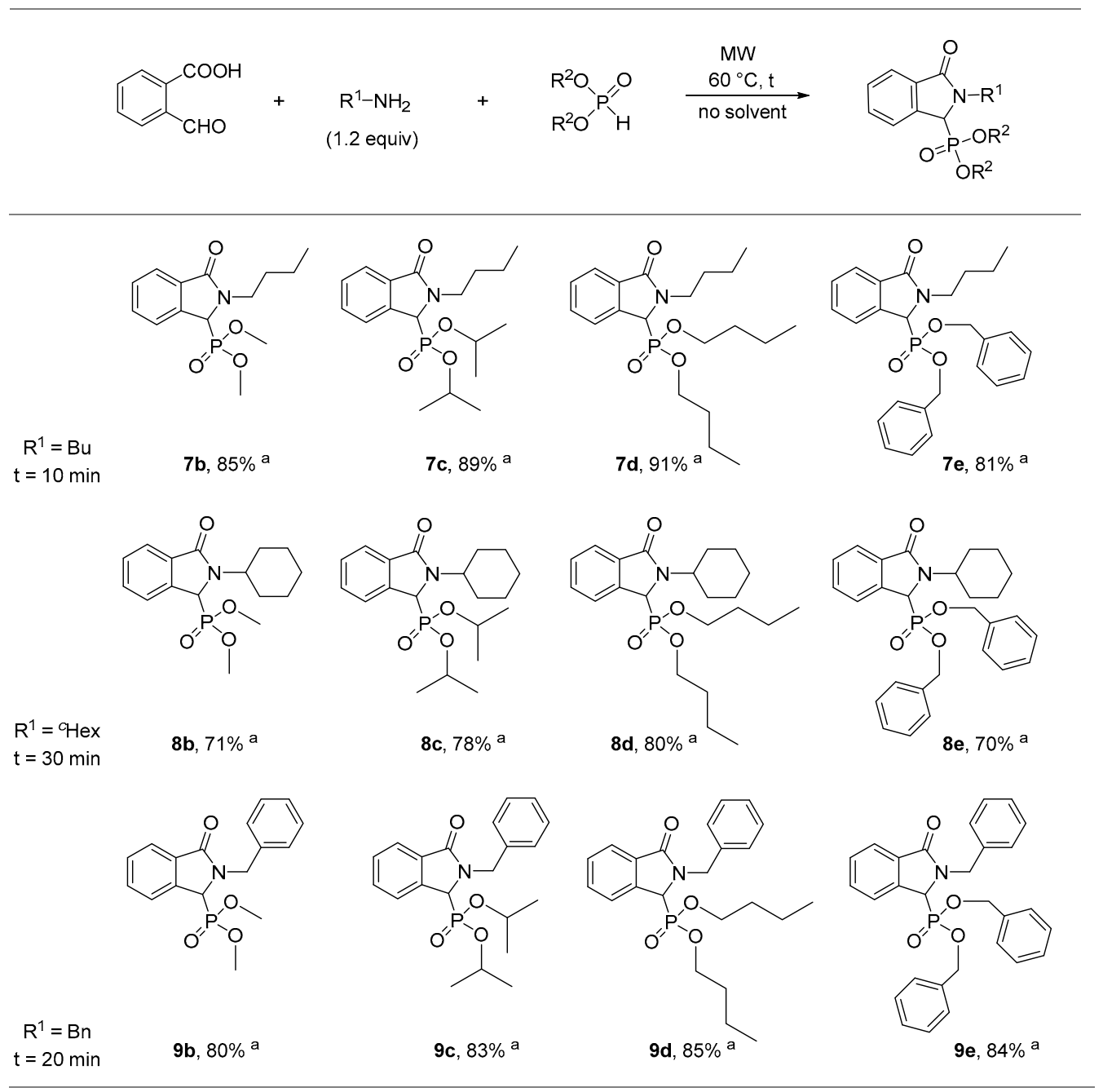

Scheme 2. Microwave (MW)-assisted condensation of 2-formylbenzoic acid, primary amines and dialkyl phosphites. ${ }^{\text {a }}$ Isolated yield.

Finally, the reaction of 2-formylbenzoic acid and butylamine was also carried out using ethyl phenyl- $\mathrm{H}$-phosphinate as the P-reagent at $60{ }^{\circ} \mathrm{C}$, for an irradiation of $10 \mathrm{~min}$ (Scheme 3). After column chromatography, product 10 was isolated in a yield of $78 \%$. Due to the chiral center on the phosphorus atom, the isoindolin-1-one phosphinate (10) was obtained as a mixture of diastereomers in a ratio of 47:53 based on ${ }^{31} \mathrm{P}$ NMR spectroscopy.

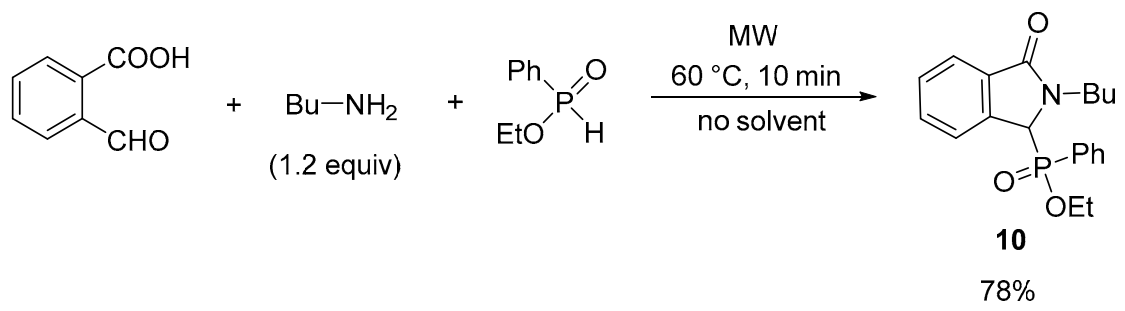

Scheme 3. Microwave-assisted condensation of 2-formylbenzoic acid, butylamine and ethyl phenyl- $H$-phosphinate.

Altogether 16 isoindolin-1-one-3-phosphonate derivatives were synthesized in high yields at low temperature for a short reaction time, and characterized by ${ }^{31} \mathrm{P},{ }^{1} \mathrm{H}$ and ${ }^{13} \mathrm{C}$ NMR spectroscopy, 
as well as by HRMS. Except products $\mathbf{9 a}$ and $\mathbf{9 b}$, all derivatives are new compounds. (Copies of ${ }^{31} \mathrm{P},{ }^{1} \mathrm{H}$, and ${ }^{13} \mathrm{C}$ NMR spectra for all compounds synthesized are presented in the Supplementary Materials.)

\subsection{Study on the Condensation of 2-Formylbenzoic Acid, Butylamine and Diethyl Phosphite by In Situ} FT-IR Spectroscopy

The condensation of 2-formylbenzoic acid, butylamine and diethyl phosphite was followed by in situ Fourier transform (FT)-IR spectroscopy (Scheme 4). The reaction was carried out under conventional heating at $60^{\circ} \mathrm{C}$ in ethanol as the solvent.

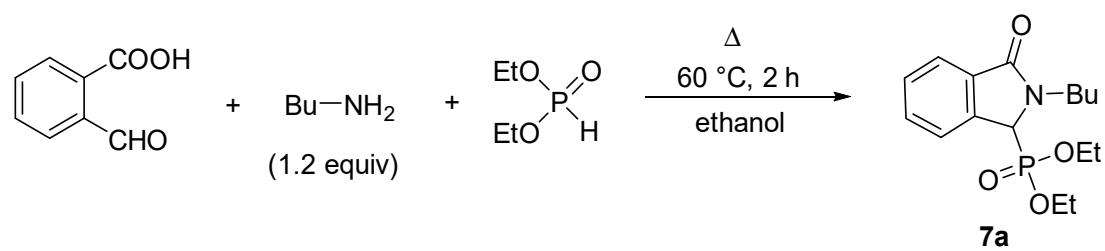

Scheme 4. Condensation of 2-formylbenzoic acid, butylamine and diethyl phosphite in ethanol.

The first step was to register the IR spectra of the reaction components in ethanol solution (Figure 2). 2-Formylbenzoic acid (FBA) has a strong absorption band at $1756 \mathrm{~cm}^{-1}$ corresponding to the $v_{\mathrm{C}=\mathrm{O}}$. The single $v_{\mathrm{C}=\mathrm{O}}$ absorption suggests that $\mathbf{F B A}$ is present in its lactone tautomer form in ethanol [30,31]. At the same time, diethyl phosphite (DEP) has strong signals at $964 \mathrm{~cm}^{-1}$ and $1254 \mathrm{~cm}^{-1}$ assigned to the $v_{\mathrm{P}-\mathrm{O}-\mathrm{C}}$ and the $v_{\mathrm{P}=\mathrm{O}}$ vibrations, respectively. Butylamine (BA) may be identified based on the $\delta_{\mathrm{C}-\mathrm{H}}\left(1381 \mathrm{~cm}^{-1}\right)$ and $\delta_{\mathrm{N}-\mathrm{H}}\left(1605 \mathrm{~cm}^{-1}\right)$ absorptions. In the spectrum of diethyl (2-butyl-3-oxo-2,3-dihydro-1H-isoindol-1-yl)phosphonate (7a), a strong characteristic $v_{\mathrm{C}=\mathrm{O}}$ signal can be observed at $1690 \mathrm{~cm}^{-1}$.

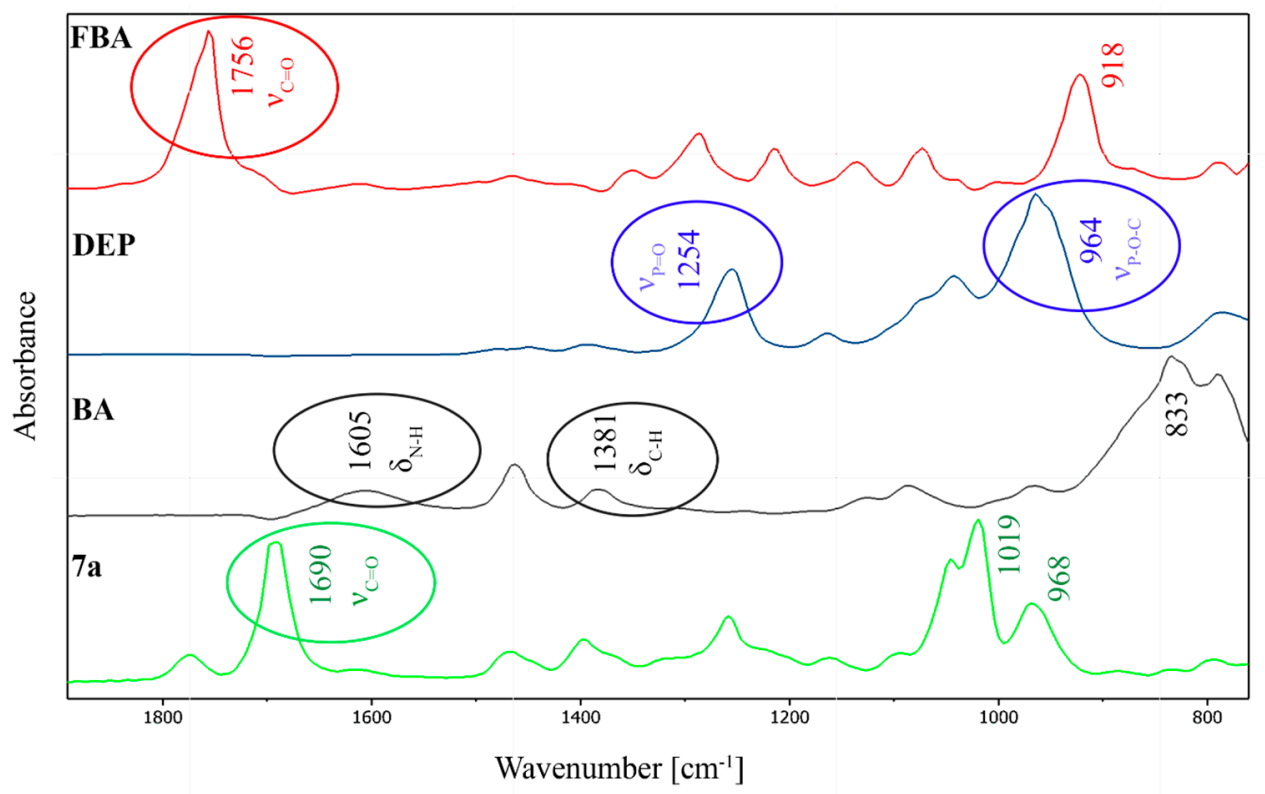

Figure 2. IR spectra of the reaction components in ethanol (absorptions of the solvent were subtracted for clarity).

In the in situ FT-IR investigation, the starting materials were mixed in ten-minute intervals in the following order: 1. FBA, 2. DEP, 3. BA. Then, the mixture was heated to $60^{\circ} \mathrm{C}$ in an oil bath and was reacted until completion. A segment of the time-dependent IR spectrum (3D diagram) can be seen in Figure 3. The reaction components (FBA, DEP, BA and 7a) could be easily identified by their characteristic absorptions (Figure 2). 


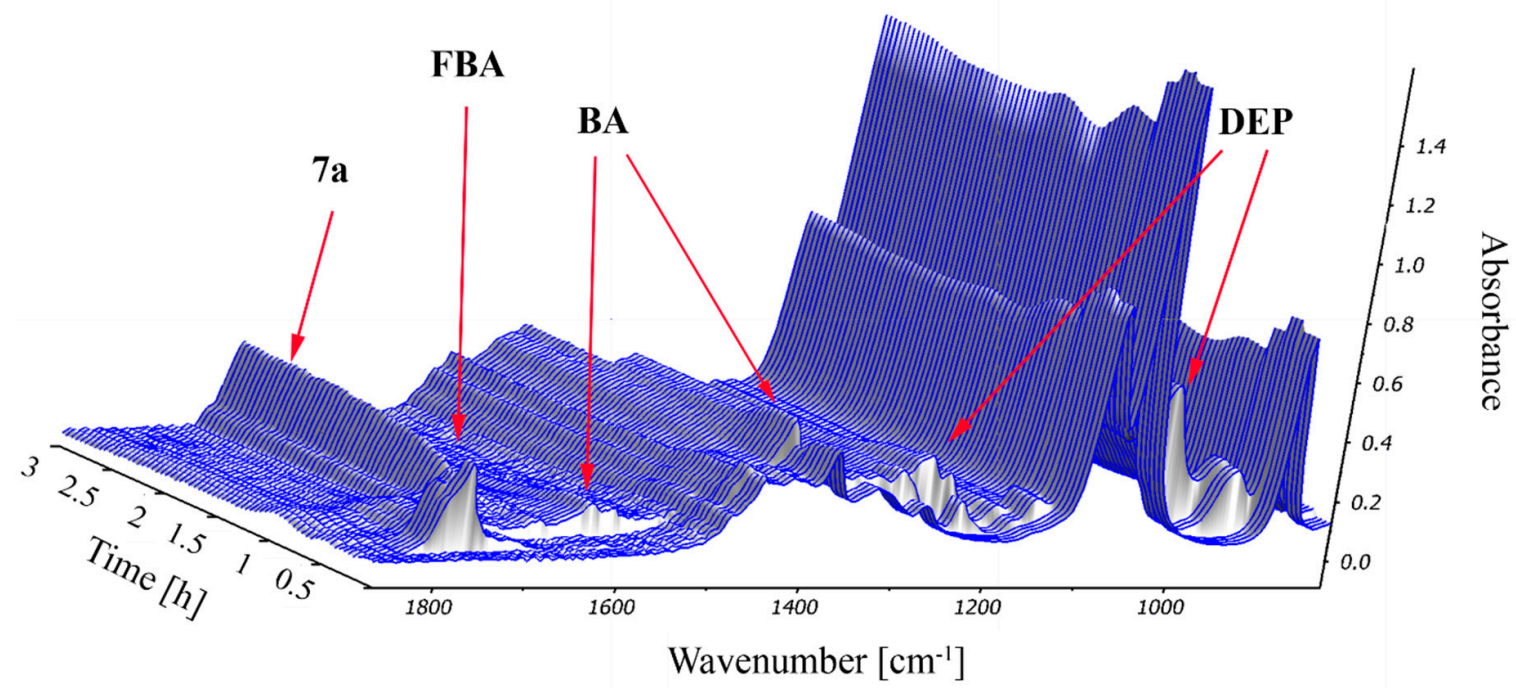

Figure 3. A segment of the time-dependent IR spectrum of the condensation of 2-formylbenzoic acid (FBA), butylamine (BA) and diethyl phosphite (DEP) in ethanol.

The result of our study is the diagram showing the concentration profile of the starting components (FBA, DEP and BA) and the product (7a) (Figure 4). This was constructed by deconvolution on the basis of the decrease/increase of the different absorptions on the time scale. This calculation (MCR-ALS, multivariate curve resolution-alternating least squares) gives the concentration profiles of the components and the spectra of pure components. It can be seen-also in the 3D diagram (Figure 3) - that the signal of FBA immediately decreased after adding BA; however, no new significant absorptions could be observed, which would indicate the formation of an intermediate, such as an imine [32]. Decreasing of the signals is possibly due to the change of IR properties of FBA in the presence of BA. During the reaction, the relative concentrations of the starting materials were constantly decreasing, while the signal of product $7 \mathbf{a}$ was constantly increasing. The condensation was complete at ca. $2 \mathrm{~h}$.

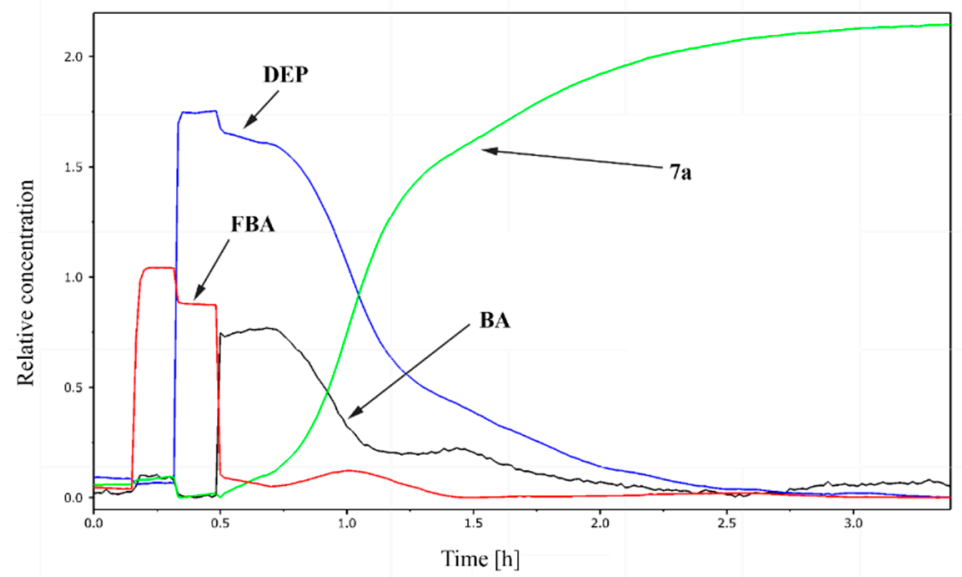

Figure 4. Concentration profiles of the reaction components in the condensation of 2-formylbenzoic acid (FBA), butylamine (BA) and diethyl phosphite (DEP) in ethanol.

Finally, the IR spectra of the reaction components (FBA, DEP, BA and 7a) were extracted from the calculation (Figure 5). As compared to the spectra obtained in ethanol solution (Figure 2), the agreement is rather good. 


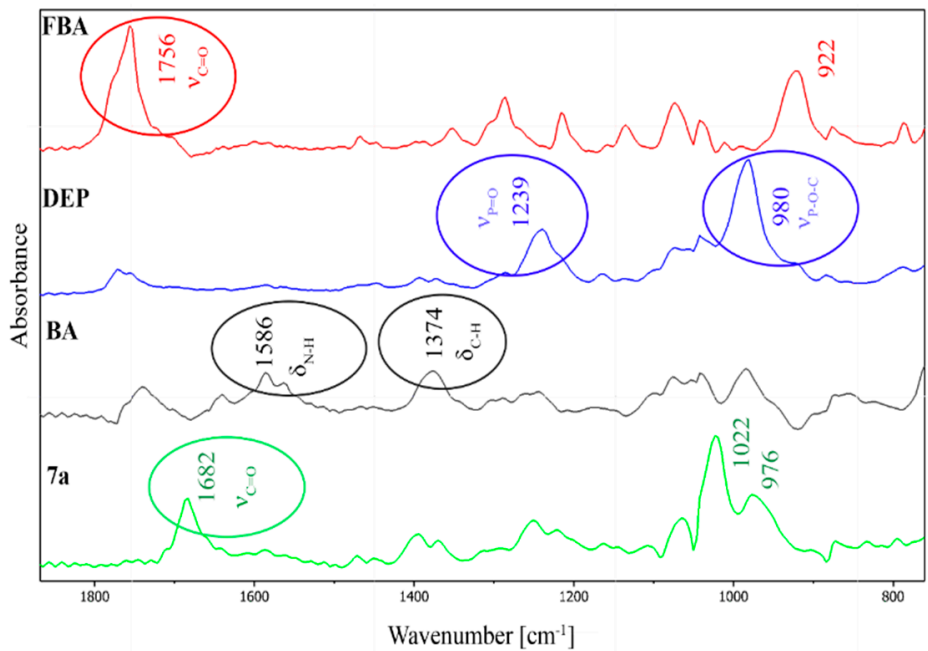

Figure 5. IR spectra of the reaction components obtained after deconvolution.

2.3. Condensation of 2-Formylbenzoic Acid, Aliphatic Primary Amines and Diethyl Phosphite in a Continuous Flow Microwave Reactor

In order to increase the productivity of the MW-assisted synthesis developed, the reaction of 2-formylbenzoic acid, aliphatic primary amines and diethyl phosphite was studied using a dual pump continuous flow system containing a CEM ${ }^{\circledR}$ (Matthews, NC, USA) MW reactor equipped with a commercially available CEM ${ }^{\circledR}$ continuous flow cell (Figures S1 and S2) (Scheme 5, Table 2).

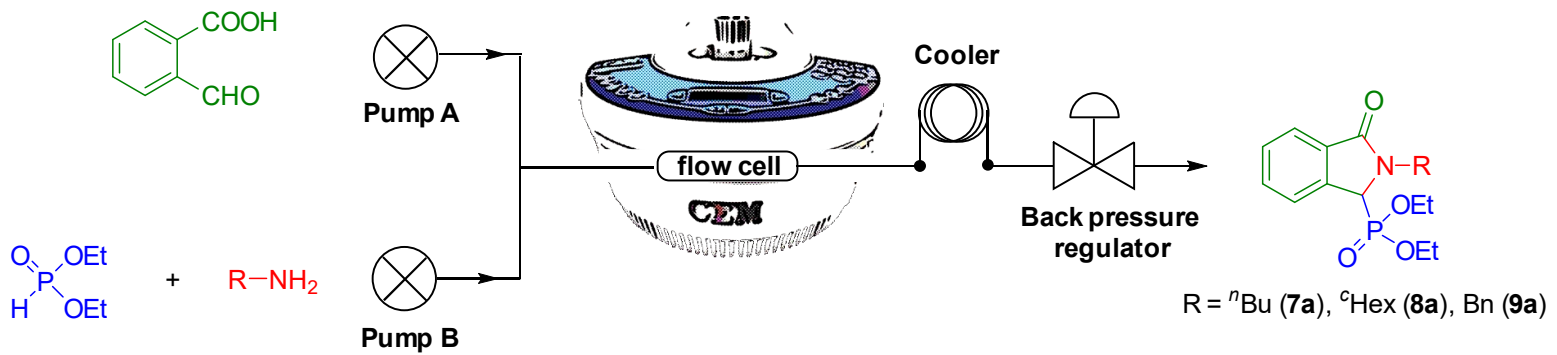

Scheme 5. Condensation of 2-formylbenzoic acid, aliphatic primary amines and diethyl phosphite in a continuous flow microwave reactor.

The 2-formylbenzoic acid in ethanol (solution A) and the mixture of the corresponding amine and diethyl phosphite in ethanol (solution B) were fed separately into the mixer. The mixture reached the reactor at a summa flow rate of $0.15-0.70 \mathrm{~mL} / \mathrm{min}$ (corresponding to residence times of $45-10 \mathrm{~min}$, respectively). The temperature was monitored and controlled by the IR sensor of the MW device. The mixture leaving the reactor was cooled down to $25^{\circ} \mathrm{C}$ using a spiral-like cooler and was passed through a back pressure regulator operating at $250 \mathrm{psi}$ ( $17 \mathrm{bar}$ ). Consecutive fractions of the leaving mixture were analyzed by GC measurements to determine the composition and to identify the stationary operation.

Performing the condensation using 1.2 equivalents of the butylamine at $60{ }^{\circ} \mathrm{C}$ at a flow rate of $0.7 \mathrm{~mL} / \mathrm{min}$ (corresponding to a residence time of $10 \mathrm{~min}$ ), the conversion was $43 \%$ (Table 2, Entry 1). Increasing the residence time to $20 \mathrm{~min}$ (by decreasing the flow rate to $0.35 \mathrm{~mL} / \mathrm{min}$ ), the proportion of the isoindolin-1-one-3-phosphonate (7a) was somewhat higher (52\%) (Table 2, Entry 2). The condensation was also carried out at a residence time of $30 \mathrm{~min}$ (at a flow rate of $0.25 \mathrm{~mL} / \mathrm{min}$ ); however, the conversion increased by only $4 \%$ (Table 2, Entry 3). In the next series of experiments, the effect of the molar ratio of the starting materials was investigated. Applying 1.5 equivalents of the butylamine at a residence time of $20 \mathrm{~min}$, the conversion already increased to $70 \%$ (Table 2, Entry 4). 
At a higher excess of butylamine (2 equivalents), the outcome did not change significantly (Table 2, Entry 5). Carrying out the condensation with 1.2 or 1.5 equivalents of diethyl phosphite, the ratio of the isoindolin-1-one-3-phosphonate (7a) was 81\% and 95\%, respectively (Table 2, Entries 6 and 7). Using 1.5 equivalents of both of the butylamine and the diethyl phosphite, the reaction was complete under a residence time of $30 \mathrm{~min}$, and the diethyl (2-butyl-3-oxo-2,3-dihydro- $1 \mathrm{H}$-isoindol-1-yl)phosphonate (7a) was isolated in a yield of $95 \%$ (Table 2, Entry 8 ).

Performing the continuous flow three-component reaction with cyclohexylamine at the same temperature $\left(60^{\circ} \mathrm{C}\right)$, the condensation was somewhat slower, as it was complete applying a residence time of $45 \mathrm{~min}$ (at a flow rate of $0.15 \mathrm{~mL} / \mathrm{min}$ ) (Table 2, Entry 9). The corresponding isoindolin-1-one-3-phosphonate (8a) was obtained in a yield of $86 \%$ after column chromatography.

In case of benzylamine, a flow rate of $0.18 \mathrm{~mL} / \mathrm{min}$ (a residence time of $40 \mathrm{~min}$ ) at $60{ }^{\circ} \mathrm{C}$ were the optimal parameters (Table 2, Entry 10). The diethyl (2-benzyl-3-oxo-2,3-dihydro$1 H$-isoindol-1-yl)phosphonate (9a) could be isolated in a yield of $91 \%$.

In the flow approaches, the productivity was $2.3 \mathrm{~g} / \mathrm{h}$ for product $7 \mathrm{a}, 1.4 \mathrm{~g} / \mathrm{h}$ for product $8 \mathrm{a}$, and $1.8 \mathrm{~g} / \mathrm{h}$ in case of compound $\mathbf{9 a}$, which are higher than the batch results $(1.8 \mathrm{~g} / \mathrm{h}, 0.6 \mathrm{~g} / \mathrm{h}$ and $1.0 \mathrm{~g} / \mathrm{h}$, respectively). However, it should be noted that the batch productivity was calculated based on the net reaction time of several (six, two and three, respectively) consecutive reactions - apart from the necessary preparations of the experiments.

Table 2. Optimization of the condensation of 2-formylbenzoic acid, aliphatic primary amines and diethyl phosphite in a continuous flow MW reactor.

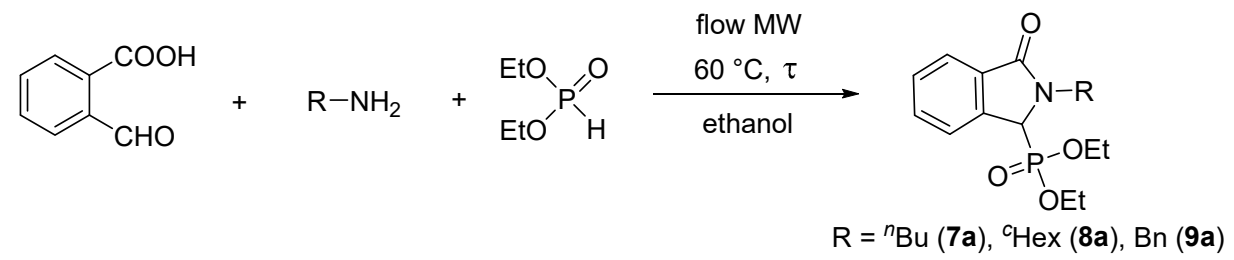

\begin{tabular}{cccccccc}
\hline Entry & $\mathbf{R}$ & $\begin{array}{c}\text { Amine } \\
\text { (equiv) }\end{array}$ & $\begin{array}{c}\text { DEP } \\
\text { (equiv) }\end{array}$ & $\begin{array}{c}\text { Flow Rate } \\
(\mathbf{m L} / \mathbf{m i n})\end{array}$ & $\boldsymbol{\tau}$ (min) & $\begin{array}{c}\text { Conversion a } \\
\mathbf{( \% )}\end{array}$ & $\begin{array}{c}\text { Yield }^{\mathbf{b}} \\
\mathbf{( \% )}\end{array}$ \\
\hline 1 & $\mathrm{Bu}$ & 1.2 & 1 & 0.70 & 10 & 43 & - \\
2 & $\mathrm{Bu}$ & 1.2 & 1 & 0.35 & 20 & 52 & - \\
3 & $\mathrm{Bu}$ & 1.2 & 1 & 0.25 & 30 & 56 & - \\
4 & $\mathrm{Bu}$ & 1.5 & 1 & 0.35 & 20 & 70 & - \\
5 & $\mathrm{Bu}$ & 2.0 & 1 & 0.35 & 20 & 73 & - \\
6 & $\mathrm{Bu}$ & 1.5 & 1.2 & 0.35 & 20 & 81 & - \\
7 & $\mathrm{Bu}$ & 1.5 & 1.5 & 0.35 & 20 & 95 & $95 \%(\mathbf{7 a})$ \\
8 & $\mathrm{Bu}$ & 1.5 & 1.5 & 0.25 & 30 & 100 & $86 \%(8 \mathbf{8})$ \\
9 & ${ }^{\circ} \mathrm{Hex}$ & 1.5 & 1.5 & 0.15 & 45 & 100 & $91 \%(\mathbf{9 a})$ \\
10 & $\mathrm{Bn}$ & 1.5 & 1.5 & 0.18 & 40 & 100 &
\end{tabular}

\section{Materials and Methods}

\subsection{General}

The reactions were carried out in a $300 \mathrm{~W}$ CEM Discover focused microwave reactor (CEM Microwave Technology Ltd., Buckingham, UK) equipped with a pressure controller using 5-20 W irradiation under isothermal conditions.

The continuous flow reactions were performed in a self-developed continuous flow system comprising a 300 W CEM Discover (CEM Microwave Technology Ltd., Buckingham, UK) focused microwave reactor equipped with a CEM $10 \mathrm{~mL}$ Flow Cell Accessory continuous flow unit (irradiated volume $7 \mathrm{~mL}$ ) [29,33,34], a Gilson 332 dual HPLC pump (Gilson U.K., Bedfordshire, UK), an HPLC backpressure regulator with a 250 Psi (17.2 bar) cartridge (CEM Microwave Technology Ltd., 
Buckingham, UK) and a cooler. Teflon ${ }^{\circledR}$ PFA tubes (DuPont, Wilmington, DE, USA) with outside diameter $0.125^{\prime \prime}(3.175 \mathrm{~mm})$ and inside diameter $0.064^{\prime \prime}(1.575 \mathrm{~mm})$ were used. The exact lengths and volumes of each tube parts are shown in Figure S1. All of the tubes, nuts and ferrules applied were fully compatible with a regular HPLC system.

GC measurements were performed on an Agilent ${ }^{\circledR} 6890$ GC-FID chromatograph (Agilent, Santa Clara, CA, USA), using a $15 \mathrm{~m} \times 0.18 \mathrm{~mm}$ Restek, Rtx-5 column with a film layer of $0.20 \mu \mathrm{m}$. The temperature of the column was initially held at $40^{\circ} \mathrm{C}$ for $1 \mathrm{~min}$, followed by programming at $25^{\circ} \mathrm{C} / \mathrm{min}$ up to $300{ }^{\circ} \mathrm{C}$ and a final period at $300^{\circ} \mathrm{C}$ (isothermal) for $10 \mathrm{~min}$. The temperature of the injector was $290^{\circ} \mathrm{C}$ and of the FID detector $300{ }^{\circ} \mathrm{C}$. The carrier gas was $\mathrm{N}_{2}$.

The ${ }^{31} \mathrm{P},{ }^{1} \mathrm{H},{ }^{13} \mathrm{C}$, NMR spectra were taken in $\mathrm{CDCl}_{3}$ solution on a Bruker AV-300 spectrometer (Bruker Scientific LLC, Billerica, MA, USA) operating at 121.5, 300 and 75.5 MHz, respectively. Chemical shifts are downfield relative to $85 \% \mathrm{H}_{3} \mathrm{PO}_{4}$ and TMS. Couplings are given in $\mathrm{Hz}$. Non-equivalence effects were observed in ${ }^{1} \mathrm{H}$ and ${ }^{13} \mathrm{C}\left\{{ }^{1} \mathrm{H}\right\}$ NMR spectra. Corresponding pairs of resonances were marked with (I) and (II), respectively.

High resolution mass spectrometric measurements were performed using a Sciex 5600+ Q-TOF mass spectrometer (AB Sciex UK Limited, Warrington, UK) in positive electrospray mode.

In situ FT IR measurements were conducted using ReactIR 1000 equipment (Mettler-Toledo Inc., Columbus, $\mathrm{OH}, \mathrm{USA}$ ). An attenuated total reflectance (ATR) diamond probe was placed in a $100 \mathrm{~mL}$ four-necked flask equipped with a dropping funnel, a condenser, a thermometer and a magnetic stirrer. The temperature was maintained by using an appropriately adjusted oil bath.

3.2. General Procedure for the Synthesis of Isoindolin-1-One-3-Phosphonates (7-9) and Isoindolin-1-One-3-Phosphinate (10)

A mixture of $1.0 \mathrm{mmol}(0.15 \mathrm{~g})$ 2-formylbenzoic acid, $1.0 \mathrm{mmol}(0.10 \mathrm{~mL}), 1.1 \mathrm{mmol}(0.11 \mathrm{~mL})$ or $1.2 \mathrm{mmol}(0,12 \mathrm{~mL})$ butylamine, $1.2 \mathrm{mmol}(0.14 \mathrm{~mL})$ cyclohexylamine or $1.2 \mathrm{mmol}(0.13 \mathrm{~mL})$ benzylamine and $>\mathrm{P}(\mathrm{O}) \mathrm{H}$ derivative $(1.0 \mathrm{mmol}$ (diethyl phosphite $(0.13 \mathrm{~mL})$, dimethyl phosphite $(0.09 \mathrm{~mL})$, diisopropyl phosphite $(0.17 \mathrm{~mL})$, dibutyl phosphite $(0.20 \mathrm{~mL})$, dibenzyl phosphite $(0.22 \mathrm{~mL})$, ethyl phenyl- $H$-phosphinate $(0.15 \mathrm{~mL})$ or $1.1 \mathrm{mmol}(0.14 \mathrm{~mL})$ or $1.2 \mathrm{mmol}(0.16 \mathrm{~mL})$ diethyl phosphite was irradiated in a sealed tube at $40-60^{\circ} \mathrm{C}$ for $10-30 \mathrm{~min}$ in a CEM Microwave reactor equipped with a pressure controller. The volatile components were removed in vacuum, and the residue was analysed by gas chromatography. The isoindolin-1-one-3-phosphonates and isoindolin-1-one-3-phosphinate were obtained by column chromatography using silica gel as the absorbent and dichloromethane:methanol (97:3) as the eluent. The following products were thus prepared:

\subsubsection{Diethyl (2-Butyl-3-oxo-2,3-dihydro- $1 H$-isoindol-1-yl)phosphonate (7a)}

Yield: 94\% (0.31 g), colourless oil; ${ }^{31} \mathrm{P}\left(\mathrm{CDCl}_{3}\right) \delta 18.3 ;{ }^{1} \mathrm{H} \mathrm{NMR}\left(\mathrm{CDCl}_{3}\right) \delta 0.94\left(\mathrm{t}, J_{\mathrm{HH}}=7.4,3 \mathrm{H}\right.$, $\left.\mathrm{CH}_{3}\left(\mathrm{CH}_{2}\right)_{3} \mathrm{~N}\right), 1.12\left(\mathrm{t}, J_{\mathrm{HH}}=7.1,3 \mathrm{H}, \mathrm{CH}_{3} \mathrm{CH}_{2} \mathrm{O}^{\mathrm{I}}\right), 1.27\left(\mathrm{t}, J_{\mathrm{HH}}=7.1,3 \mathrm{H}, \mathrm{CH}_{3} \mathrm{CH}_{2} \mathrm{O}^{\mathrm{II}}\right), 1.29-1.38(\mathrm{~m}, 2 \mathrm{H}$, $\left.\mathrm{CH}_{2}\left(\mathrm{CH}_{2}\right)_{2} \mathrm{~N}\right), 1.56-1.65\left(\mathrm{~m}, 1 \mathrm{H}, \mathrm{CH}_{\mathrm{A}}, \mathrm{CH}_{2} \mathrm{CH}_{2} \mathrm{~N}\right), 1.65-1.75\left(\mathrm{~m}, 1 \mathrm{H}, \mathrm{CH}_{\mathrm{B}}, \mathrm{CH}_{2} \mathrm{CH}_{2} \mathrm{~N}\right), 3.53-3.60(\mathrm{~m}, 1 \mathrm{H}$, $\left.\mathrm{CH}_{\mathrm{A}}, \mathrm{CH}_{2} \mathrm{~N}\right), 3.76-3.85\left(\mathrm{~m}, 1 \mathrm{H}, \mathrm{CH}_{\mathrm{A}}, \mathrm{CH}_{2} \mathrm{O}^{\mathrm{I}}\right), 3.91-4.00\left(\mathrm{~m}, 1 \mathrm{H}, \mathrm{CH}_{\mathrm{B}}, \mathrm{CH}_{2} \mathrm{O}^{\mathrm{I}}\right), 4.08-4.18\left(\mathrm{~m}, 3 \mathrm{H}, \mathrm{CH}_{\mathrm{B}}\right.$, $\left.\mathrm{CH}_{2} \mathrm{~N}, \mathrm{CH}_{2} \mathrm{O}^{\mathrm{II}}\right), 4.89\left(\mathrm{~d}, J_{\mathrm{HP}}=13.6,1 \mathrm{H}, \mathrm{C}_{1} \mathrm{H}\right), 7.51\left(\mathrm{t}, J_{\mathrm{HH}}=7.5,1 \mathrm{H}, \mathrm{C}_{5} \mathrm{H}\right), 7.58\left(\mathrm{t}, J_{\mathrm{HH}}=8.0,1 \mathrm{H}, \mathrm{C} 6 \mathrm{H}\right)$, $7.78\left(\mathrm{~d}, J_{\mathrm{HH}}=7.6,1 \mathrm{H}, \mathrm{C}_{7} \mathrm{H}\right), 7.87\left(\mathrm{~d}, J_{\mathrm{HH}}=7.5,1 \mathrm{H}, \mathrm{C}_{4} \mathrm{H}\right) ;{ }^{13} \mathrm{C} \mathrm{NMR}\left(\mathrm{CDCl}_{3}\right) \delta 13.8\left(\mathrm{CH}_{3}\left(\mathrm{CH}_{2}\right)_{3} \mathrm{~N}\right)$, $16.2\left(\mathrm{~d},{ }^{3} J_{\mathrm{CP}}=5.6, \mathrm{CH}_{3} \mathrm{CH}_{2} \mathrm{O}^{\mathrm{I}}\right), 16.4\left(\mathrm{~d},{ }^{3} J_{\mathrm{CP}}=5.6, \mathrm{CH}_{3} \mathrm{CH}_{2} \mathrm{O}^{\mathrm{II}}\right), 20.0\left(\mathrm{CH}_{2}\left(\mathrm{CH}_{2}\right)_{2} \mathrm{~N}\right), 30.0\left(\mathrm{CH}_{2} \mathrm{CH}_{2} \mathrm{~N}\right)$, $41.5\left(\mathrm{CH}_{2} \mathrm{~N}\right), 57.2\left(\mathrm{~d},{ }^{1} J_{\mathrm{CP}}=155.0, \mathrm{C}_{1}\right), 63.2\left(\mathrm{~d},{ }^{2} J_{\mathrm{CP}}=7.3, \mathrm{CH}_{2} \mathrm{O}^{\mathrm{I}}\right), 63.5\left(\mathrm{~d},{ }^{2} J_{\mathrm{CP}}=7.1, \mathrm{CH}_{2} \mathrm{O}^{\mathrm{II}}\right)$, $123.8\left(\mathrm{~d}, J_{\mathrm{CP}}=1.6, \mathrm{C}_{4}\right), 124.5\left(\mathrm{~d},{ }^{3} J_{\mathrm{CP}}=2.6, \mathrm{C}_{7}\right), 128.9\left(\mathrm{~d}, J_{\mathrm{CP}}=2.2, \mathrm{C}_{5}\right), 131.5\left(\mathrm{~d}, J_{\mathrm{CP}}=2.5, \mathrm{C}_{6}\right)$, $132.5\left(\mathrm{~d},{ }^{3} J_{\mathrm{CP}}=4.3, \mathrm{C}_{3 \mathrm{a}}\right), 138.5\left(\mathrm{~d},{ }^{2} J_{\mathrm{CP}}=5.8, \mathrm{C}_{7 \mathrm{a}}\right), 168.8\left(\mathrm{~d},{ }^{3} J_{\mathrm{CP}}=3.5, \mathrm{C}_{3}\right) ;[\mathrm{M}+\mathrm{H}]^{+}$found $=326.1509$, $\mathrm{C}_{16} \mathrm{H}_{25} \mathrm{NO}_{4} \mathrm{P}$ requires 326.1521 .

\subsubsection{Dimethyl (2-Butyl-3-oxo-2,3-dihydro-1H-isoindol-1-yl)phosphonate (7b)}

Yield: $85 \%$ (0.25 g), white crystals; Mp: $77-78{ }^{\circ} \mathrm{C} ;{ }^{31} \mathrm{P}\left(\mathrm{CDCl}_{3}\right) \delta 18.5 ;{ }^{1} \mathrm{H} \mathrm{NMR}\left(\mathrm{CDCl}_{3}\right)$ $\delta 0.94\left(\mathrm{t}, J_{\mathrm{HH}}=7.3,3 \mathrm{H}, \mathrm{CH}_{3}\left(\mathrm{CH}_{2}\right)_{3} \mathrm{~N}\right), 1.22-1.45\left(\mathrm{~m}, 2 \mathrm{H}, \mathrm{CH}_{2}\left(\mathrm{CH}_{2}\right)_{2} \mathrm{~N}\right), 1.49-1.81\left(\mathrm{~m}, 2 \mathrm{H}, \mathrm{CH}_{2} \mathrm{CH}_{2} \mathrm{~N}\right)$, 
3.42-3.64 [3.56 $\left(\mathrm{d}, \mathrm{J}_{\mathrm{HH}}=10.9, \mathrm{CH}_{3} \mathrm{O}^{\mathrm{I}}\right)$ overlapped by the multiplet of $\mathrm{CH}_{\mathrm{A}}, \mathrm{CH}_{2} \mathrm{~N}$ total int. $4 \mathrm{H}$ ], $3.75\left(\mathrm{~d}, J_{\mathrm{HH}}=10.9,3 \mathrm{H}, \mathrm{CH}_{3} \mathrm{O}^{\mathrm{II}}\right), 4.03-4.24\left(\mathrm{~m}, 1 \mathrm{H}, \mathrm{CH}_{\mathrm{B}}, \mathrm{CH}_{2} \mathrm{~N}\right), 4.91\left(\mathrm{~d}, J_{\mathrm{HP}}=13.4,1 \mathrm{H}, \mathrm{C} 1 \mathrm{H}\right)$, $7.52\left(\mathrm{t}, J_{\mathrm{HH}}=6.9,1 \mathrm{H}, \mathrm{C}_{5} \mathrm{H}\right), 7.59\left(\mathrm{t}, J_{\mathrm{HH}}=6.4,1 \mathrm{H}, \mathrm{C}_{6} \mathrm{H}\right), 7.77\left(\mathrm{~d}, J_{\mathrm{HH}}=7.5,1 \mathrm{H}, \mathrm{C}_{7} \mathrm{H}\right), 7.88\left(\mathrm{~d}, J_{\mathrm{HH}}=7.4\right.$, $\left.1 \mathrm{H}, \mathrm{C}_{4} \mathrm{H}\right) ;{ }^{13} \mathrm{C}$ NMR $\left(\mathrm{CDCl}_{3}\right) \delta 13.7\left(\mathrm{CH}_{3}\left(\mathrm{CH}_{2}\right)_{3} \mathrm{~N}\right), 19.9\left(\mathrm{CH}_{2}\left(\mathrm{CH}_{2}\right)_{2} \mathrm{~N}\right), 29.9\left(\mathrm{CH}_{2} \mathrm{CH}_{2} \mathrm{~N}\right), 41.5\left(\mathrm{CH}_{2} \mathrm{~N}\right)$, $53.6\left(\mathrm{~d},{ }^{2} J_{\mathrm{CP}}=7.3, \mathrm{CH}_{3} \mathrm{O}^{\mathrm{I}}\right), 53.7\left(\mathrm{~d},{ }^{2} J_{\mathrm{CP}}=7.1, \mathrm{CH}_{3} \mathrm{O}^{\mathrm{II}}\right), 56.7\left(\mathrm{~d},{ }^{1} J_{\mathrm{CP}}=155.4, \mathrm{C}_{1}\right), 123.8\left(\mathrm{~d}, J_{\mathrm{CP}}=1.6\right.$, $\left.\mathrm{C}_{4}\right), 124.3\left(\mathrm{~d},{ }^{3} J_{\mathrm{CP}}=2.7, \mathrm{C}_{7}\right), 128.9\left(\mathrm{~d}, J_{\mathrm{CP}}=2.2, \mathrm{C}_{5}\right), 131.5\left(\mathrm{~d}, J_{\mathrm{CP}}=2.5, \mathrm{C}_{6}\right), 132.3\left(\mathrm{~d},{ }^{3} J_{\mathrm{CP}}=4.1\right.$, $\left.\mathrm{C}_{3 \mathrm{a}}\right), 138.2\left(\mathrm{~d},{ }^{2} J_{\mathrm{CP}}=5.9, \mathrm{C}_{7 \mathrm{a}}\right), 168.6\left(\mathrm{~d},{ }^{3} J_{\mathrm{CP}}=3.4, \mathrm{C}_{3}\right) ;[\mathrm{M}+\mathrm{H}]^{+}$found $=298.1196, \mathrm{C}_{14} \mathrm{H}_{21} \mathrm{NO}_{4} \mathrm{P}$ requires 298.1208 .

\subsubsection{Diisopropyl (2-Butyl-3-oxo-2,3-dihydro- $1 H$-isoindol-1-yl)phosphonate (7c)}

Yield: 89\% (0.32 g), colourless oil; ${ }^{31} \mathrm{P}\left(\mathrm{CDCl}_{3}\right) \delta 16.6 ;{ }^{1} \mathrm{H} \mathrm{NMR}\left(\mathrm{CDCl}_{3}\right) \delta 0.92\left(\mathrm{t}, J_{\mathrm{HH}}=7.3,3 \mathrm{H}\right.$, $\left.\mathrm{CH}_{3}\left(\mathrm{CH}_{2}\right)_{3} \mathrm{~N}\right), 0.95\left(\mathrm{~d}, J_{\mathrm{HH}}=7.3,3 \mathrm{H}, \mathrm{CH}_{3} \mathrm{CH}^{\mathrm{I}}\right), 1.19-1.41\left[1.24\left(\mathrm{~d}, J_{\mathrm{HH}}=6.2, \mathrm{CH}_{3} \mathrm{CH}^{\mathrm{II}}\right), 1.25\left(\mathrm{~d}, J_{\mathrm{HH}}=6.2\right.\right.$, $\left.\mathrm{CH}_{3} \mathrm{CH}^{\mathrm{III}}\right), 1.28\left(\mathrm{~d}, \mathrm{~J}_{\mathrm{HH}}=6.1, \mathrm{CH}_{3} \mathrm{CH}^{\mathrm{IV}}\right)$ overlapped by the multiplet of $\mathrm{CH}_{2}\left(\mathrm{CH}_{2}\right)_{2} \mathrm{~N}$ total int. $\left.11 \mathrm{H}\right]$, 1.52-1.79 (m, $\left.2 \mathrm{H}, \mathrm{CH}_{2} \mathrm{CH}_{2} \mathrm{~N}\right), 3.51-3.65\left(\mathrm{~m}, 1 \mathrm{H}, \mathrm{CH}_{\mathrm{A}}, \mathrm{CH}_{2} \mathrm{~N}\right), 4.06-4.20\left(\mathrm{~m}, 1 \mathrm{H}, \mathrm{CH}_{\mathrm{B}}, \mathrm{CH}_{2} \mathrm{~N}\right)$, $4.32-4.49\left(\mathrm{~m}, 1 \mathrm{H}, \mathrm{CHO}^{\mathrm{I}}\right), 4.66-4.80\left(\mathrm{~m}, 1 \mathrm{H}, \mathrm{CHO}^{\mathrm{II}}\right), 4.84\left(\mathrm{~d}, J_{\mathrm{HP}}=13.8,1 \mathrm{H}, \mathrm{C}_{1} \mathrm{H}\right), 7.50\left(\mathrm{t}, J_{\mathrm{HH}}=7.3,1 \mathrm{H}\right.$, $\left.\mathrm{C}_{5} \mathrm{H}\right), 7.56\left(\mathrm{t}, J_{\mathrm{HH}}=6.8,1 \mathrm{H}, \mathrm{C}_{6} \mathrm{H}\right), 7.79\left(\mathrm{~d}, J_{\mathrm{HH}}=7.5,1 \mathrm{H}, \mathrm{C}_{7} \mathrm{H}\right), 7.86\left(\mathrm{~d}, J_{\mathrm{HH}}=7.2,1 \mathrm{H}, \mathrm{C}_{4} \mathrm{H}\right) ;{ }^{13} \mathrm{C} \mathrm{NMR}$ $\left(\mathrm{CDCl}_{3}\right) \delta 13.8\left(\mathrm{CH}_{3}\left(\mathrm{CH}_{2}\right)_{3} \mathrm{~N}\right), 20.0\left(\mathrm{CH}_{2}\left(\mathrm{CH}_{2}\right)_{2} \mathrm{~N}\right), 23.3\left(\mathrm{~d},{ }^{3} J_{\mathrm{CP}}=5.3, \mathrm{CH}_{3} \mathrm{CH}^{\mathrm{I}}\right), 23.9\left(\mathrm{~d},{ }^{3} J_{\mathrm{CP}}=5.0\right.$, $\left.\mathrm{CH}_{3} \mathrm{CH}^{\mathrm{II}}\right), 24.06\left(\mathrm{~d},{ }^{3} \mathrm{~J}_{\mathrm{CP}}=6.6, \mathrm{CH}_{3} \mathrm{CH}^{\mathrm{III}}\right), 24.10\left(\mathrm{~d},{ }^{3} J_{\mathrm{CP}}=6.1, \mathrm{CH}_{3} \mathrm{CH}^{\mathrm{IV}}\right), 30.0\left(\mathrm{CH}_{2} \mathrm{CH}_{2} \mathrm{~N}\right), 41.5\left(\mathrm{CH}_{2} \mathrm{~N}\right)$, $57.1\left(\mathrm{~d},{ }^{1} J_{\mathrm{CP}}=155.0, \mathrm{C}_{1}\right), 63.1\left(\mathrm{~d},{ }^{2} J_{\mathrm{CP}}=7.3, \mathrm{CHO}^{\mathrm{I}}\right), 63.4\left(\mathrm{~d},{ }^{2} J_{\mathrm{CP}}=7.1, \mathrm{CHO}^{\mathrm{II}}\right), 123.7\left(\mathrm{~d}, J_{\mathrm{CP}}=1.6\right.$, $\left.\mathrm{C}_{4}\right), 124.4\left(\mathrm{~d},{ }^{3} J_{\mathrm{CP}}=2.6, \mathrm{C}_{7}\right), 128.8\left(\mathrm{~d}, J_{\mathrm{CP}}=2.2, \mathrm{C}_{5}\right), 131.4\left(\mathrm{~d}, J_{\mathrm{CP}}=2.5, \mathrm{C}_{6}\right), 132.4\left(\mathrm{~d},{ }^{3} J_{\mathrm{CP}}=4.3\right.$, $\left.\left.\mathrm{C}_{3 \mathrm{a}}\right), 138.4\left(\mathrm{~d},{ }^{2} J_{\mathrm{CP}}=5.8, \mathrm{C}_{7 \mathrm{a}}\right), 168.7\left(\mathrm{~d},{ }^{3} J_{\mathrm{CP}}=3.5, \mathrm{C}_{3}\right) ; \mathrm{M}+\mathrm{H}\right]^{+}$found $=354.1826, \mathrm{C}_{18} \mathrm{H}_{29} \mathrm{NO}_{4} \mathrm{P}$ requires 354.1828 .

\subsubsection{Dibutyl (2-Butyl-3-oxo-2,3-dihydro-1H-isoindol-1-yl)phosphonate (7d)}

Yield: $91 \%(0.35 \mathrm{~g})$, colourless oil; ${ }^{31} \mathrm{P}\left(\mathrm{CDCl}_{3}\right) \delta 18.3 ;{ }^{1} \mathrm{H} \mathrm{NMR}\left(\mathrm{CDCl}_{3}\right) \delta 0.82\left(\mathrm{t}, J_{\mathrm{HH}}=7.3\right.$, $\left.3 \mathrm{H}, \mathrm{CH}_{3}\left(\mathrm{CH}_{2}\right)_{3} \mathrm{O}^{\mathrm{I}}\right), 0.89\left(\mathrm{t}, J_{\mathrm{HH}}=7.2,3 \mathrm{H}, \mathrm{CH}_{3}\left(\mathrm{CH}_{2}\right)_{3} \mathrm{O}^{\mathrm{II}}\right), 0.94\left(\mathrm{t}, J_{\mathrm{HH}}=7.3,3 \mathrm{H}, \mathrm{CH}_{3}\left(\mathrm{CH}_{2}\right)_{3} \mathrm{~N}\right)$, 1.13-1.49 (m, 8H, CH $\left.\left(\mathrm{CH}_{2}\right)_{2} \mathrm{~N}, \mathrm{CH}_{2}\left(\mathrm{CH}_{2}\right)_{2} \mathrm{O}, \mathrm{CH}_{2} \mathrm{CH}_{2} \mathrm{O}^{\mathrm{I}}\right), 1.49-1.79\left(\mathrm{~m}, 4 \mathrm{H}, \mathrm{CH}_{2} \mathrm{CH}_{2} \mathrm{~N}, \mathrm{CH}_{2} \mathrm{CH}_{2} \mathrm{O}^{\mathrm{II}}\right)$, 3.46-3.62 (m, $\left.1 \mathrm{H}, \mathrm{CH}_{\mathrm{A}}, \mathrm{CH}_{2} \mathrm{~N}\right), 3.63-3.78\left(\mathrm{~m}, 1 \mathrm{H}, \mathrm{CH}_{\mathrm{A}}, \mathrm{CH}_{2} \mathrm{O}^{\mathrm{I}}\right), 3.81-3.95\left(\mathrm{~m}, 1 \mathrm{H}, \mathrm{CH}_{\mathrm{B}}, \mathrm{CH}_{2} \mathrm{O}^{\mathrm{I}}\right)$, 3.96-4.24 (m, 3H, $\left.\mathrm{CH}_{\mathrm{B}}, \mathrm{CH}_{2} \mathrm{~N}, \mathrm{CH}_{2} \mathrm{O}^{\mathrm{II}}\right), 4.89\left(\mathrm{~d}, J_{\mathrm{HP}}=13.6,1 \mathrm{H}, \mathrm{C}_{1} \mathrm{H}\right), 7.51\left(\mathrm{t}, J_{\mathrm{HH}}=7.4,1 \mathrm{H}, \mathrm{C}_{5} \mathrm{H}\right)$, $7.57\left(\mathrm{t}, J_{\mathrm{HH}}=7.4,1 \mathrm{H}, \mathrm{C}_{6} \mathrm{H}\right), 7.78\left(\mathrm{~d}, J_{\mathrm{HH}}=7.5,1 \mathrm{H}, \mathrm{C}_{7} \mathrm{H}\right), 7.86\left(\mathrm{~d}, J_{\mathrm{HH}}=7.3,1 \mathrm{H}, \mathrm{C}_{4} \mathrm{H}\right) ;{ }^{13} \mathrm{C} \mathrm{NMR}$ $\left(\mathrm{CDCl}_{3}\right) \delta 13.41\left(\mathrm{CH}_{3}\left(\mathrm{CH}_{2}\right)_{3} \mathrm{O}^{\mathrm{I}}\right), 13.43\left(\mathrm{CH}_{3}\left(\mathrm{CH}_{2}\right)_{3} \mathrm{O}^{\mathrm{II}}\right), 13.7\left(\mathrm{CH}_{3}\left(\mathrm{CH}_{2}\right)_{3} \mathrm{~N}\right), 18.5\left(\mathrm{CH}_{2}\left(\mathrm{CH}_{2}\right)_{2} \mathrm{O}^{\mathrm{I}}\right), 18.6$ $\left(\mathrm{CH}_{2}\left(\mathrm{CH}_{2}\right)_{2} \mathrm{O}^{\mathrm{II}}\right), 20.0\left(\mathrm{CH}_{2}\left(\mathrm{CH}_{2}\right)_{2} \mathrm{~N}\right), 30.0\left(\mathrm{CH}_{2} \mathrm{CH}_{2} \mathrm{~N}\right), 32.3\left(\mathrm{~d},{ }^{3} J_{\mathrm{CP}}=5.6, \mathrm{CH}_{2} \mathrm{CH}_{2} \mathrm{O}^{\mathrm{I}}\right), 32.4\left(\mathrm{~d},{ }^{3} J_{\mathrm{CP}}=5.9\right.$, $\left.\mathrm{CH}_{2} \mathrm{CH}_{2} \mathrm{O}^{\mathrm{II}}\right), 41.5\left(\mathrm{CH}_{2} \mathrm{~N}\right), 57.1\left(\mathrm{~d},{ }^{1} J_{\mathrm{CP}}=155.0, \mathrm{C}_{1}\right), 66.7\left(\mathrm{~d},{ }^{2} J_{\mathrm{CP}}=7.5, \mathrm{CH}_{2} \mathrm{O}^{\mathrm{I}}\right), 67.1\left(\mathrm{~d},{ }^{2} J_{\mathrm{CP}}=7.2\right.$, $\left.\mathrm{CH}_{2} \mathrm{O}^{\mathrm{II}}\right), 123.7\left(\mathrm{~d}, J_{\mathrm{CP}}=1.4, \mathrm{C}_{4}\right), 124.4\left(\mathrm{~d},{ }^{3} J_{\mathrm{CP}}=2.7, \mathrm{C}_{7}\right), 128.8\left(\mathrm{~d}, J_{\mathrm{CP}}=2.2, \mathrm{C}_{5}\right), 131.4\left(\mathrm{~d}, J_{\mathrm{CP}}=2.4, \mathrm{C}_{6}\right)$, $132.5\left(\mathrm{~d},{ }^{3} J_{\mathrm{CP}}=4.3, \mathrm{C}_{3 \mathrm{a}}\right), 138.5\left(\mathrm{~d},{ }^{2} J_{\mathrm{CP}}=5.8, \mathrm{C}_{7 \mathrm{a}}\right), 168.6\left(\mathrm{~d},{ }^{3} J_{\mathrm{CP}}=3.3, \mathrm{C}_{3}\right) ;[\mathrm{M}+\mathrm{H}]^{+}$found $=382.2131$, $\mathrm{C}_{20} \mathrm{H}_{33} \mathrm{NO}_{4} \mathrm{P}$ requires 382.2147.

\subsubsection{Dibenzyl (2-Butyl-3-oxo-2,3-dihydro-1H-isoindol-1-yl)phosphonate (7e)}

Yield: $81 \%$ (0.36 g), white crystals; Mp: $103-104{ }^{\circ} \mathrm{C} ;{ }^{31} \mathrm{P}\left(\mathrm{CDCl}_{3}\right) \delta 19.2 ;{ }^{1} \mathrm{H} \mathrm{NMR}\left(\mathrm{CDCl}_{3}\right)$ $\delta 0.85\left(\mathrm{t}, J_{\mathrm{HH}}=7.3,3 \mathrm{H}, \mathrm{CH}_{3}\left(\mathrm{CH}_{2}\right)_{3} \mathrm{~N}\right), 1.13-1.30\left(\mathrm{~m}, 2 \mathrm{H}, \mathrm{CH}_{2}\left(\mathrm{CH}_{2}\right)_{2} \mathrm{~N}\right), 1.41-1.65\left(\mathrm{~m}, 2 \mathrm{H}, \mathrm{CH}_{2} \mathrm{CH}_{2} \mathrm{~N}\right)$, 3.37-3.56 (m, 1H, $\left.\mathrm{CH}_{\mathrm{A}}, \mathrm{CH}_{2} \mathrm{~N}\right), 3.96-4.14\left(\mathrm{~m}, 1 \mathrm{H}, \mathrm{CH}_{\mathrm{B}}, \mathrm{CH}_{2} \mathrm{~N}\right), 4.61-4.76\left(\mathrm{~m}, 1 \mathrm{H}, \mathrm{C}_{1} \mathrm{H}\right), 4.76-4.90(\mathrm{~m}, 2 \mathrm{H}$, $\left.\mathrm{CH}_{2} \mathrm{O}^{\mathrm{I}}\right), 4.90-5.06\left(\mathrm{~m}, 2 \mathrm{H}, \mathrm{CH}_{2} \mathrm{O}^{\mathrm{II}}\right), 7.10-7.24\left(\mathrm{~m}, 4 \mathrm{H}, \mathrm{C}_{2^{\prime}} \mathrm{H}\right), 7.24-7.57\left(\mathrm{~m}, 6 \mathrm{H}, \mathrm{C}_{3,4^{\prime}} \mathrm{H}\right), 7.43-7.57(\mathrm{~m}, 2 \mathrm{H}$, $\left.\mathrm{C}_{5} \mathrm{H}, \mathrm{C}_{6} \mathrm{H}\right), 7.73\left(\mathrm{~d}, \mathrm{~J}_{\mathrm{HH}}=7.2,1 \mathrm{H}, \mathrm{C}_{7} \mathrm{H}\right), 7.81\left(\mathrm{~d}, \mathrm{~J}_{\mathrm{HH}}=7.0,1 \mathrm{H}, \mathrm{C}_{4} \mathrm{H}\right) ;{ }^{13} \mathrm{C} \mathrm{NMR}\left(\mathrm{CDCl}_{3}\right) \delta 13.7$ $\left(\mathrm{CH}_{3}\left(\mathrm{CH}_{2}\right)_{3} \mathrm{~N}\right), 19.9\left(\mathrm{CH}_{2}\left(\mathrm{CH}_{2}\right)_{2} \mathrm{~N}\right), 29.9\left(\mathrm{CH}_{2} \mathrm{CH}_{2} \mathrm{~N}\right), 41.5\left(\mathrm{CH}_{2} \mathrm{~N}\right), 57.4\left(\mathrm{~d},{ }^{1} J_{\mathrm{CP}}=154.9, \mathrm{C}_{1}\right)$, $68.6\left(\mathrm{~d},{ }^{2} J_{\mathrm{CP}}=7.3, \mathrm{CH}_{2} \mathrm{O}^{\mathrm{I}}\right), 68.8\left(\mathrm{~d},{ }^{2} J_{\mathrm{CP}}=7.0, \mathrm{CH}_{2} \mathrm{O}^{\mathrm{II}}\right), 123.8\left(\mathrm{~d}, J_{\mathrm{CP}}=1.6, \mathrm{C}_{4}\right), 124.4\left(\mathrm{~d},{ }^{3} J_{\mathrm{CP}}=2.7\right.$, $\left.\mathrm{C}_{7}\right), 128.1\left(\mathrm{C}_{3^{\prime}}{ }^{\mathrm{I}}\right), 128.2\left(\mathrm{C}_{3^{\prime}}{ }^{\prime I}\right), 128.66\left(\mathrm{C}_{2^{\prime}}{ }^{\mathrm{I}}\right), 128.67\left(\mathrm{C}_{2^{\prime}}{ }^{\mathrm{II}}\right), 128.87\left(\mathrm{C}_{4^{\prime}}{ }^{\mathrm{I}}\right), 128.90\left(\mathrm{C}_{4^{\prime}}{ }^{\mathrm{II}}\right), 128.9\left(\mathrm{~d}, J_{\mathrm{CP}}=2.4\right.$, $\left.\mathrm{C}_{5}\right), 131.4\left(\mathrm{~d}, J_{\mathrm{CP}}=2.5, \mathrm{C}_{6}\right), 132.5\left(\mathrm{~d},{ }^{3} J_{\mathrm{CP}}=4.2, \mathrm{C}_{3 \mathrm{a}}\right), 135.43\left(\mathrm{~d},{ }^{3} J_{\mathrm{CP}}=5.5, \mathrm{C}_{1^{\prime}}{ }^{\mathrm{I}}\right), 135.44\left(\mathrm{~d},{ }^{3} J_{\mathrm{CP}}=5.7\right.$, $\left.\mathrm{C}_{1^{\prime}}{ }^{\mathrm{II}}\right), 138.2\left(\mathrm{~d},{ }^{2} J_{\mathrm{CP}}=6.0, \mathrm{C}_{7 \mathrm{a}}\right), 168.6\left(\mathrm{~d},{ }^{3} J_{\mathrm{CP}}=3.5, \mathrm{C}_{3}\right) ;[\mathrm{M}+\mathrm{H}]^{+}$found $=450.1822, \mathrm{C}_{26} \mathrm{H}_{29} \mathrm{NO}_{4} \mathrm{P}$ requires 450.1828 . 


\subsubsection{Diethyl (2-Cyclohexyl-3-oxo-2,3-dihydro-1H-isoindol-1-yl)phosphonate (8a)}

Yield: $84 \%(0.30 \mathrm{~g})$, colourless oil; ${ }^{31} \mathrm{P}\left(\mathrm{CDCl}_{3}\right) \delta 18.7 ;{ }^{1} \mathrm{H} \mathrm{NMR}\left(\mathrm{CDCl}_{3}\right) \delta 1.14\left(\mathrm{t}, J_{\mathrm{HP}}=7.1,3 \mathrm{H}\right.$, $\left(\mathrm{CH}_{3} \mathrm{CH}_{2} \mathrm{O}^{\mathrm{I}}\right), 1.28-1.45\left[1.25\left(\mathrm{t}, \mathrm{J}_{\mathrm{HH}}=7.1, \mathrm{CH}_{3} \mathrm{CH}_{2} \mathrm{O}^{\mathrm{II}}\right)\right.$ overlapped by the multiplet of ${ }^{c} \mathrm{HexH}$ total int. $6 \mathrm{H}], 1.57-2.03\left(\mathrm{~m}, 5 \mathrm{H},{ }^{c} \mathrm{HexH}\right), 2.13-2.31\left(\mathrm{~m}, 1 \mathrm{H},{ }^{c} \mathrm{HexH}\right), 2.36-2.55\left(\mathrm{~m}, 1 \mathrm{H},{ }^{c} \mathrm{HexH}\right), 3.69-3.88(\mathrm{~m}, 2 \mathrm{H}$, $\left.\mathrm{CH}_{2} \mathrm{O}^{\mathrm{I}}\right), 3.88-4.02\left(\mathrm{~m}, 1 \mathrm{H}, \mathrm{C}_{1^{\prime}} \mathrm{H}\right), 4.02-4.19\left(\mathrm{~m}, 2 \mathrm{H}, \mathrm{CH}_{2} \mathrm{O}^{\mathrm{II}}\right), 4.85\left(\mathrm{~d}, 1 \mathrm{H}, J_{\mathrm{HP}}=13.2, \mathrm{C}_{1} \mathrm{H}\right), 7.49(\mathrm{t}, 1 \mathrm{H}$, $\left.J_{\mathrm{HH}}=7.6, \mathrm{C}_{5} \mathrm{H}\right), 7.55\left(\mathrm{t}, 1 \mathrm{H}, J_{\mathrm{HH}}=7.2, \mathrm{C}_{6} \mathrm{H}\right), 7.74\left(\mathrm{~d}, J_{\mathrm{HH}}=7.6,1 \mathrm{H}, \mathrm{C}_{7} \mathrm{H}\right), 7.82\left(\mathrm{~d}, J_{\mathrm{HH}}=7.3,1 \mathrm{H}, \mathrm{C}_{4} \mathrm{H}\right)$; ${ }^{13} \mathrm{C}$ NMR $\left(\mathrm{CDCl}_{3}\right) \delta 16.2\left(\mathrm{~d},{ }^{3} J_{\mathrm{CP}}=4.6, \mathrm{CH}_{3} \mathrm{CH}_{2} \mathrm{O}^{\mathrm{I}}\right), 16.3\left(\mathrm{~d},{ }^{3} J_{\mathrm{CP}}=5.5, \mathrm{CH}_{3} \mathrm{CH}_{2} \mathrm{O}^{\mathrm{II}}\right), 25.3\left(\mathrm{C}_{4^{\prime}}\right), 26.1$ $\left(\mathrm{C}_{3^{\prime}}{ }^{\mathrm{I}}\right), 26.3\left(\mathrm{C}_{3^{\prime}}{ }^{\mathrm{II}}\right), 29.3\left(\mathrm{C}_{2^{\prime}}{ }^{\mathrm{I}}\right), 29.7\left(\mathrm{C}_{2^{\prime}}{ }^{\mathrm{II}}\right), 56.7\left(\mathrm{C}_{1^{\prime}}\right), 58.6\left(\mathrm{~d},{ }^{1} J_{\mathrm{CP}}=155.4, \mathrm{C}_{1}\right), 63.2\left(\mathrm{~d},{ }^{2} J_{\mathrm{CP}}=7.5, \mathrm{CH}_{2} \mathrm{O}^{\mathrm{I}}\right)$, $63.4\left(\mathrm{~d},{ }^{2} J_{\mathrm{CP}}=7.0, \mathrm{CH}_{2} \mathrm{O}^{\mathrm{II}}\right), 123.4\left(\mathrm{~d}, J_{\mathrm{CP}}=1.3, \mathrm{C}_{4}\right), 124.4\left(\mathrm{~d},{ }^{3} J_{\mathrm{CP}}=2.7, \mathrm{C}_{7}\right), 128.7\left(\mathrm{~d}, J_{\mathrm{CP}}=2.3, \mathrm{C}_{5}\right)$, $131.2\left(\mathrm{~d}, J_{\mathrm{CP}}=2.6, \mathrm{C}_{6}\right), 133.6\left(\mathrm{~d},{ }^{3} J_{\mathrm{CP}}=4.0, \mathrm{C}_{3 \mathrm{a}}\right), 138.8\left(\mathrm{~d},{ }^{2} J_{\mathrm{CP}}=5.7, \mathrm{C}_{7 \mathrm{a}}\right), 169.0\left(\mathrm{~d},{ }^{3} J_{\mathrm{CP}}=3.4, \mathrm{C}_{3}\right)$; $[\mathrm{M}+\mathrm{H}]^{+}$found $=352.1672, \mathrm{C}_{18} \mathrm{H}_{27} \mathrm{NO}_{4} \mathrm{P}$ requires 352.1672 .

\subsubsection{Dimethyl (2-Cyclohexyl-3-oxo-2,3-dihydro-1H-isoindol-1-yl)phosphonate (8b)}

Yield: $71 \%$ (0.23 g), colourless oil; ${ }^{31} \mathrm{P}\left(\mathrm{CDCl}_{3}\right) \delta 20.9 ;{ }^{1} \mathrm{H}$ NMR $\left(\mathrm{CDCl}_{3}\right) 1.18-1.45\left(\mathrm{~m}, 3 \mathrm{H},{ }^{c} \mathrm{HexH}\right)$, 1.60-1.72 (m, 1H, $\left.{ }^{c} \mathrm{HexH}\right), 1.72-2.02\left(\mathrm{~m}, 4 \mathrm{H},{ }^{c} \mathrm{HexH}\right), 2.10-2.30\left(\mathrm{~m}, 1 \mathrm{H},{ }^{c} \mathrm{HexH}\right), 2.31-2.50\left(\mathrm{~m}, 1 \mathrm{H},{ }^{c} \mathrm{HexH}\right)$, $3.59\left(\mathrm{~d}, J_{\mathrm{HP}}=10.7,3 \mathrm{H}, \mathrm{CH}_{3} \mathrm{O}^{\mathrm{I}}\right), 3.76-3.86\left[3.72\left(\mathrm{~d}, J_{\mathrm{HP}}=10.9, \mathrm{CH}_{3} \mathrm{O}^{\mathrm{II}}\right)\right.$ overlapped by the multiplet of $\mathrm{C}_{1^{\prime}} \mathrm{H}$ total int. $\left.4 \mathrm{H}\right], 4.88\left(\mathrm{~d}, J_{\mathrm{HP}}=13.1,1 \mathrm{H}, \mathrm{C}_{1} \mathrm{H}\right), 7.44-7.61\left(\mathrm{~m}, 2 \mathrm{H}, \mathrm{C}_{5} \mathrm{H}, \mathrm{C}_{6} \mathrm{H}\right), 7.71\left(\mathrm{~d}, J_{\mathrm{HH}}=7.5,1 \mathrm{H}\right.$, $\left.\mathrm{C}_{7} \mathrm{H}\right), 7.83\left(\mathrm{~d}, J_{\mathrm{HH}}=7.2,1 \mathrm{H}, \mathrm{C}_{4} \mathrm{H}\right) ;{ }^{13} \mathrm{C} \mathrm{NMR}\left(\mathrm{CDCl}_{3}\right) \delta 25.2\left(\mathrm{C}_{4^{\prime}}\right), 26.0\left(\mathrm{C}_{3^{\prime}}{ }^{\mathrm{I}}\right), 26.3\left(\mathrm{C}_{3^{\prime}}{ }^{\mathrm{II}}\right), 29.4\left(\mathrm{C}_{2^{\prime}}{ }^{\mathrm{I}}\right)$, $29.7\left(\mathrm{C}_{2^{\prime}}{ }^{\mathrm{II}}\right), 53.7\left(\mathrm{~d},{ }^{2} J_{\mathrm{CP}}=7.2, \mathrm{CH}_{3} \mathrm{O}^{\mathrm{I}}\right), 53.8\left(\mathrm{~d},{ }^{2} J_{\mathrm{CP}}=7.2, \mathrm{CH}_{3} \mathrm{O}^{\mathrm{II}}\right), 56.6\left(\mathrm{C}_{1^{\prime}}\right), 59.2\left(\mathrm{~d},{ }^{1} J_{\mathrm{CP}}=156.4\right.$, $\left.\mathrm{C}_{1}\right), 123.5\left(\mathrm{~d}, J_{\mathrm{CP}}=1.6, \mathrm{C}_{4}\right), 124.3\left(\mathrm{~d},{ }^{3} J_{\mathrm{CP}}=2.6, \mathrm{C}_{7}\right), 128.8\left(\mathrm{~d}, J_{\mathrm{CP}}=2.2, \mathrm{C}_{5}\right), 131.3\left(\mathrm{~d}, J_{\mathrm{CP}}=2.5, \mathrm{C}_{6}\right)$, $133.4\left(\mathrm{~d},{ }^{3} J_{\mathrm{CP}}=4.1, \mathrm{C}_{3 \mathrm{a}}\right), 138.5\left(\mathrm{~d},{ }^{2} J_{\mathrm{CP}}=6.0, \mathrm{C}_{7 \mathrm{a}}\right), 169.0\left(\mathrm{~d},{ }^{3} J_{\mathrm{CP}}=3.5, \mathrm{C}_{3}\right) ;[\mathrm{M}+\mathrm{H}]^{+}$found $=324.1364$, $\mathrm{C}_{16} \mathrm{H}_{23} \mathrm{NO}_{4} \mathrm{P}$ requires 324.1359 .

\subsubsection{Diisopropyl (2-Cyclohexyl-3-oxo-2,3-dihydro-1H-isoindol-1-yl)phosphonate (8c)}

Yield: $78 \%(0.30 \mathrm{~g})$, white crystals; Mp: ${ }^{112-113}{ }^{\circ} \mathrm{C} ;{ }^{31} \mathrm{P}\left(\mathrm{CDCl}_{3}\right) \delta 17.1 ;{ }^{1} \mathrm{H}$ NMR $\left(\mathrm{CDCl}_{3}\right)$ $\delta 0.96\left(\mathrm{~d}, J_{\mathrm{HH}}=6.2,3 \mathrm{H}, \mathrm{CH}_{3} \mathrm{CH}^{\mathrm{I}}\right), 1.15-1.44\left(1.23\left[\mathrm{~d}, J_{\mathrm{HH}}=6.2, \mathrm{CH}_{3} \mathrm{CH}^{\mathrm{II}}\right), 1.26\left(\mathrm{~d}, J_{\mathrm{HH}}=6.1\right.\right.$, $\left.\mathrm{CH}_{3} \mathrm{CH}^{\mathrm{III}}, \mathrm{CH}_{3} \mathrm{CH}^{\mathrm{IV}}\right)$ overlapped by the multiplet of ${ }^{c} \mathrm{HexH}$ total int. $\left.12 \mathrm{H}\right], 1.57-2.03\left(\mathrm{~m}, 5 \mathrm{H},{ }^{c} \mathrm{HexH}\right)$, 2.16-2.36 (m, 1H, $\left.{ }^{c} \mathrm{HexH}\right), 2.38-2.59\left(\mathrm{~m}, 1 \mathrm{H},{ }^{c} \mathrm{HexH}\right), 3.68-3.88\left(\mathrm{~m}, 1 \mathrm{H}, \mathrm{C}_{1^{\prime}} \mathrm{H}\right), 4.30-4.49\left(\mathrm{~m}, 1 \mathrm{H}, \mathrm{CHO}^{\mathrm{I}}\right)$, 4.64-4.85 [4.79 $\left(\mathrm{d}, J_{\mathrm{HP}}=13.5, \mathrm{C}_{1} \mathrm{H}\right)$ overlapped by the multiplet of $\mathrm{CHO}^{\mathrm{II}}$ total int. $\left.2 \mathrm{H}\right], 7.42-7.58$ [7.47 $\left(\mathrm{t}, J_{\mathrm{HH}}=7.4, \mathrm{C}_{5} \mathrm{H}\right)$ overlapped by the multiplet of $\mathrm{C}_{6} \mathrm{H}$ total int. $\left.2 \mathrm{H}\right], 7.75\left(\mathrm{~d}, J_{\mathrm{HH}}=7.6,1 \mathrm{H}\right.$, $\left.\mathrm{C}_{7} \mathrm{H}\right), 7.81\left(\mathrm{~d}, J_{\mathrm{HH}}=7.2,1 \mathrm{H}, \mathrm{C}_{4} \mathrm{H}\right) ;{ }^{13} \mathrm{C} \mathrm{NMR}\left(\mathrm{CDCl}_{3}\right) \delta 23.4\left(\mathrm{~d},{ }^{3} J_{\mathrm{CP}}=5.3, \mathrm{CH}_{3} \mathrm{CH}^{\mathrm{I}}\right), 23.9\left(\mathrm{~d},{ }^{3} J_{\mathrm{CP}}=5.3\right.$, $\left.\mathrm{CH}_{3} \mathrm{CH}^{\mathrm{II}}\right), 24.0\left(\mathrm{~d},{ }^{3} J_{\mathrm{CP}}=3.5, \mathrm{CH}_{3} \mathrm{CH}^{\mathrm{III}}\right), 24.1\left(\mathrm{~d},{ }^{3} J_{\mathrm{CP}}=3.4, \mathrm{CH}_{3} \mathrm{CH}^{\mathrm{IV}}\right), 25.2\left(\mathrm{C}_{4^{\prime}}\right), 26.1\left(\mathrm{C}_{3^{\prime}}{ }^{\mathrm{I}}\right), 26.3\left(\mathrm{C}_{3^{\prime}}{ }^{\mathrm{II}}\right)$, $29.2\left(\mathrm{C}_{2^{\prime}}{ }^{\mathrm{I}}\right), 29.6\left(\mathrm{C}_{2^{\prime}}{ }^{\mathrm{II}}\right), 56.7\left(\mathrm{C}_{1^{\prime}}\right), 59.2\left(\mathrm{~d}^{1}{ }^{1} J_{\mathrm{CP}}=156.3, \mathrm{C}_{1}\right), 72.0\left(\mathrm{~d},{ }^{2} J_{\mathrm{CP}}=7.7, \mathrm{CHO}{ }^{\mathrm{I}}\right), 72.4\left(\mathrm{~d}^{2} J_{\mathrm{CP}}=7.3\right.$, $\left.\mathrm{CHO}^{\mathrm{II}}\right), 123.2\left(\mathrm{~d}, J_{\mathrm{CP}}=1.7, \mathrm{C}_{4}\right), 124.5\left(\mathrm{~d},{ }^{3} J_{\mathrm{CP}}=2.6, \mathrm{C}_{7}\right), 128.6\left(\mathrm{~d}, J_{\mathrm{CP}}=2.2, \mathrm{C}_{5}\right), 131.1\left(\mathrm{~d}, J_{\mathrm{CP}}=2.6, \mathrm{C}_{6}\right)$, $133.8\left(\mathrm{~d},{ }^{3} J_{\mathrm{CP}}=4.1, \mathrm{C}_{3 \mathrm{a}}\right), 139.0\left(\mathrm{~d},{ }^{2} J_{\mathrm{CP}}=5.6, \mathrm{C}_{7 \mathrm{a}}\right), 169.0\left(\mathrm{~d},{ }^{3} J_{\mathrm{CP}}=3.7, \mathrm{C}_{3}\right) ;[\mathrm{M}+\mathrm{H}]^{+}$found $=380.1990$, $\mathrm{C}_{20} \mathrm{H}_{31} \mathrm{NO}_{4} \mathrm{P}$ requires 380.1985 .

\subsubsection{Dibutyl (2-Cyclohexyl-3-oxo-2,3-dihydro-1H-isoindol-1-yl)phosphonate (8d)}

Yield: $80 \%(0.33 \mathrm{~g})$, colourless oil; ${ }^{31} \mathrm{P}\left(\mathrm{CDCl}_{3}\right) \delta 20.6 ;{ }^{1} \mathrm{H} \mathrm{NMR}\left(\mathrm{CDCl}_{3}\right) \delta 0.85\left(\mathrm{t}, J_{\mathrm{HH}}=7.3\right.$, $\left.3 \mathrm{H}, \mathrm{CH}_{3}\left(\mathrm{CH}_{2}\right)_{3} \mathrm{O}^{\mathrm{I}}\right), 0.86\left(\mathrm{t}, J_{\mathrm{HH}}=7.3,3 \mathrm{H}, \mathrm{CH}_{3}\left(\mathrm{CH}_{2}\right)_{3} \mathrm{O}^{\mathrm{II}}\right), 1.17-1.36\left(\mathrm{~m}, 6 \mathrm{H}, \mathrm{CH}_{2}\left(\mathrm{CH}_{2}\right)_{2} \mathrm{O},{ }^{c} \mathrm{HexH}\right)$, 1.39-1.49 (m, 2H, $\left.\mathrm{CH}_{2} \mathrm{CH}_{2} \mathrm{O}^{\mathrm{I}}\right), 1.49-1.60\left(\mathrm{~m}, 2 \mathrm{H}, \mathrm{CH}_{2} \mathrm{CH}_{2} \mathrm{O}^{\mathrm{II}}\right), 1.62-1.98\left(\mathrm{~m}, 6 \mathrm{H},{ }^{c} \mathrm{HexH}\right), 2.15-2.33(\mathrm{~m}, 1 \mathrm{H}$, $\left.{ }^{c} \mathrm{HexH}\right), 2.35-2.54\left(\mathrm{~m}, 1 \mathrm{H},{ }^{c} \mathrm{HexH}\right), 3.64-3.82\left(\mathrm{~m}, 2 \mathrm{H}, \mathrm{CH}_{\mathrm{A}}, \mathrm{CH}_{2} \mathrm{O}^{\mathrm{I}}, \mathrm{C}_{1^{\prime}} \mathrm{H}\right), 3.82-3.95\left(\mathrm{~m}, 1 \mathrm{H}, \mathrm{CH}_{\mathrm{B}}\right.$, $\left.\mathrm{CH}_{2} \mathrm{O}^{\mathrm{I}}\right), 3.95-4.12\left(\mathrm{~m}, 2 \mathrm{H}, \mathrm{CH}_{2} \mathrm{O}^{\mathrm{II}}\right), 4.85\left(\mathrm{~d}, J_{\mathrm{HP}}=13.2,1 \mathrm{H}, \mathrm{C}_{1} \mathrm{H}\right), 7.43-7.58\left(\mathrm{~m}, 2 \mathrm{H}, \mathrm{C}_{5} \mathrm{H}, \mathrm{C}_{6} \mathrm{H}\right)$, $7.73\left(\mathrm{~d}, J_{\mathrm{HH}}=7.5,1 \mathrm{H}, \mathrm{C}_{7} \mathrm{H}\right), 7.82\left(\mathrm{~d}, J_{\mathrm{HH}}=7.2,1 \mathrm{H}, \mathrm{C}_{4} \mathrm{H}\right) ;{ }^{13} \mathrm{C} \mathrm{NMR}\left(\mathrm{CDCl}_{3}\right) \delta 13.4\left(\mathrm{CH}_{3}\left(\mathrm{CH}_{2}\right)_{3} \mathrm{O}^{\mathrm{I}}\right)$, $13.5\left(\mathrm{CH}_{3}\left(\mathrm{CH}_{2}\right)_{3} \mathrm{O}^{\mathrm{II}}\right), 18.5\left(\mathrm{CH}_{2}\left(\mathrm{CH}_{2}\right)_{2} \mathrm{O}^{\mathrm{I}}\right), 18.6\left(\mathrm{CH}_{2}\left(\mathrm{CH}_{2}\right)_{2} \mathrm{O}^{\mathrm{II}}\right), 25.3\left(\mathrm{C}_{4^{\prime}}\right), 26.1\left(\mathrm{C}_{3^{\prime}}{ }^{\mathrm{I}}\right), 26.3\left(\mathrm{C}_{3^{\prime}}{ }^{\mathrm{II}}\right)$, $29.3\left(\mathrm{C}_{2^{\prime}}{ }^{\mathrm{I}}\right), 29.6\left(\mathrm{C}_{2^{\prime}}{ }^{\mathrm{II}}\right), 32.36\left(\mathrm{~d},{ }^{3} J_{\mathrm{CP}}=5.6, \mathrm{CH}_{2} \mathrm{CH}_{2} \mathrm{O}^{\mathrm{I}}\right), 32.40\left(\mathrm{~d},{ }^{3} J_{\mathrm{CP}}=6.0, \mathrm{CH}_{2} \mathrm{CH}_{2} \mathrm{O}^{\mathrm{II}}\right), 56.7\left(\mathrm{C}_{1^{\prime}}\right)$, $58.6\left(\mathrm{~d},{ }^{1} J_{\mathrm{CP}}=155.6, \mathrm{C}_{1}\right), 66.9\left(\mathrm{~d},{ }^{2} J_{\mathrm{CP}}=7.6, \mathrm{CH}_{2} \mathrm{O}^{\mathrm{I}}\right), 67.1\left(\mathrm{~d},{ }^{2} J_{\mathrm{CP}}=7.3, \mathrm{CH}_{2} \mathrm{O}^{\mathrm{II}}\right), 123.3\left(\mathrm{~d}, J_{\mathrm{CP}}=1.4\right.$, $\left.\mathrm{C}_{4}\right), 124.4\left(\mathrm{~d},{ }^{3} J_{\mathrm{CP}}=2.6, \mathrm{C}_{7}\right), 128.7\left(\mathrm{~d}, J_{\mathrm{CP}}=2.2, \mathrm{C}_{5}\right), 131.1\left(\mathrm{~d}, J_{\mathrm{CP}}=2.6, \mathrm{C}_{6}\right), 133.6\left(\mathrm{~d},{ }^{3} J_{\mathrm{CP}}=4.0\right.$, 
$\left.\mathrm{C}_{3 \mathrm{a}}\right), 138.9\left(\mathrm{~d},{ }^{2} J_{\mathrm{CP}}=5.8, \mathrm{C}_{7 \mathrm{a}}\right), 169.0\left(\mathrm{~d},{ }^{3} J_{\mathrm{CP}}=3.6, \mathrm{C}_{3}\right) ;[\mathrm{M}+\mathrm{H}]^{+}$found $=408.2298, \mathrm{C}_{22} \mathrm{H}_{35} \mathrm{NO}_{4} \mathrm{P}$ requires 408.2298 .

\subsubsection{Dibenzyl (2-Cyclohexyl-3-oxo-2,3-dihydro-1H-isoindol-1-yl)phosphonate (8e)}

Yield: $70 \%(0.33 \mathrm{~g})$, white crystals; Mp: $97-98{ }^{\circ} \mathrm{C} ;{ }^{31} \mathrm{P}\left(\mathrm{CDCl}_{3}\right) \delta 19.5 ;{ }^{1} \mathrm{H} \mathrm{NMR}\left(\mathrm{CDCl}_{3}\right)$

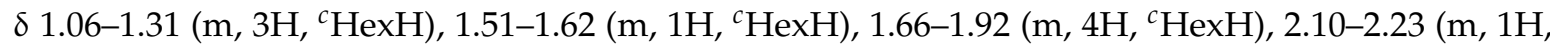
$\left.{ }^{c} \mathrm{HexH}\right), 2.30-2.42\left(\mathrm{~m}, 1 \mathrm{H},{ }^{c} \mathrm{HexH}\right), 3.67-3.77\left(\mathrm{~m}, 1 \mathrm{H}, \mathrm{C}_{1^{\prime}} \mathrm{H}\right), 4.64-4.73\left(\mathrm{~m}, 1 \mathrm{H}, \mathrm{CH}_{\mathrm{A}}, \mathrm{CH}_{2} \mathrm{O}^{\mathrm{I}}\right), 4.79-4.87$ [4.84 $\left(\mathrm{d}, J_{\mathrm{HP}}=12.7, \mathrm{C}_{1} \mathrm{H}\right)$ overlapped by the multiplet of $\mathrm{CH}_{\mathrm{B}}, \mathrm{CH}_{2} \mathrm{O}^{\mathrm{I}}$ total int. $\left.2 \mathrm{H}\right], 4.87-5.02(\mathrm{~m}, 2 \mathrm{H}$, $\left.\mathrm{CH}_{2} \mathrm{O}^{\mathrm{II}}\right), 7.14-7.21\left(\mathrm{~m}, 4 \mathrm{H}, \mathrm{C}_{2^{\prime \prime}} \mathrm{H}\right), 7.27-7.33\left(\mathrm{~m}, 6 \mathrm{H}, \mathrm{C}_{3,4^{\prime \prime}} \mathrm{H}\right), 7.45\left(\mathrm{t}, J_{\mathrm{HH}}=7.4,1 \mathrm{H}, \mathrm{C}_{5} \mathrm{H}\right), 7.50\left(\mathrm{t}, J_{\mathrm{HH}}=7.5\right.$, $\left.1 \mathrm{H}, \mathrm{C}_{6} \mathrm{H}\right), 7.69\left(\mathrm{~d}, J_{\mathrm{HH}}=8.3,1 \mathrm{H}, \mathrm{C}_{7} \mathrm{H}\right), 7.76\left(\mathrm{~d}, J_{\mathrm{HH}}=7.5,1 \mathrm{H}, \mathrm{C}_{4} \mathrm{H}\right) ;{ }^{13} \mathrm{C} \mathrm{NMR}\left(\mathrm{CDCl}_{3}\right) \delta 25.1\left(\mathrm{C}_{4^{\prime}}\right), 25.9$ $\left(\mathrm{C}_{3^{\prime}}{ }^{\mathrm{I}}\right), 26.1\left(\mathrm{C}_{3^{\prime}}{ }^{\mathrm{II}}\right), 29.3\left(\mathrm{C}_{2^{\prime}}{ }^{\mathrm{I}}\right), 29.6\left(\mathrm{C}_{2^{\prime}}{ }^{\mathrm{II}}\right), 56.5\left(\mathrm{C}_{1^{\prime}}\right), 58.8\left(\mathrm{~d},{ }^{1} J_{\mathrm{CP}}=155.4, \mathrm{C}_{1}\right), 68.7\left(\mathrm{~d},{ }^{2} J_{\mathrm{CP}}=7.5, \mathrm{CH}_{2} \mathrm{O}^{\mathrm{I}}\right)$, $68.8\left(\mathrm{~d},{ }^{2} J_{\mathrm{CP}}=6.9, \mathrm{CH}_{2} \mathrm{O}^{\mathrm{II}}\right), 123.4\left(\mathrm{~d}, J_{\mathrm{CP}}=1.4, \mathrm{C}_{4}\right), 124.3\left(\mathrm{~d},{ }^{3} J_{\mathrm{CP}}=2.4, \mathrm{C}_{7}\right), 128.0\left(\mathrm{C}_{3^{\prime \prime}}{ }^{\mathrm{I}}\right), 128.2\left(\mathrm{C}_{3^{\prime \prime}}{ }^{\mathrm{II}}\right)$, $128.58\left(\mathrm{C}_{2^{\prime \prime}} \mathrm{I}\right), 128.61\left(\mathrm{C}_{2^{\prime \prime}}{ }^{\prime \prime} \mathrm{II}\right), 128.62\left(\mathrm{C}_{4^{\prime \prime}}{ }^{\prime \prime}\right), 128.66\left(\mathrm{C}_{4^{\prime \prime}}{ }^{\prime \prime} \mathrm{II}\right), 128.74\left(\mathrm{~d}, J_{\mathrm{CP}}=2.4, \mathrm{C}_{5}\right), 131.2\left(\mathrm{~d}, J_{\mathrm{CP}}=2.5\right.$, $\left.\mathrm{C}_{6}\right), 133.5\left(\mathrm{~d},{ }^{3} J_{\mathrm{CP}}=4.3, \mathrm{C}_{3 \mathrm{a}}\right), 135.4\left(\mathrm{~d},{ }^{3} J_{\mathrm{CP}}=6.3, \mathrm{C}_{1^{\prime \prime}} \mathrm{I}\right), 135.5\left(\mathrm{~d},{ }^{3} J_{\mathrm{CP}}=5.6, \mathrm{C}_{1^{\prime \prime}}{ }^{\mathrm{II}}\right), 138.5\left(\mathrm{~d},{ }^{2} J_{\mathrm{CP}}=6.0\right.$, $\left.\mathrm{C}_{7 \mathrm{a}}\right), 168.9\left(\mathrm{~d},{ }^{3} J_{\mathrm{CP}}=3.9, \mathrm{C}_{3}\right) ;[\mathrm{M}+\mathrm{H}]^{+}$found $=476.1997, \mathrm{C}_{28} \mathrm{H}_{31} \mathrm{NO}_{4} \mathrm{P}$ requires 476.1985.

\subsubsection{Diethyl (2-Benzyl-3-oxo-2,3-dihydro-1H-isoindol-1-yl)phosphonate (9a)}

Yield: $90 \%(0.32 \mathrm{~g})$, colourless oil; ${ }^{31} \mathrm{P}\left(\mathrm{CDCl}_{3}\right) \delta 18.3 ;{ }^{31} \mathrm{P}\left(\mathrm{CDCl}_{3}\right), \delta[17] 17.7 ;[\mathrm{M}+\mathrm{H}]^{+}$found $=$ $360.1360, \mathrm{C}_{19} \mathrm{H}_{23} \mathrm{NO}_{4} \mathrm{P}$ requires 360.1359 .

\subsubsection{Dimethyl (2-Benzyl-3-oxo-2,3-dihydro- $1 H$-isoindol-1-yl)phosphonate (9b)}

Yield: $80 \%$ (0.27 g), light yellow crystals; Mp: $130-131^{\circ} \mathrm{C}$; $\mathrm{Mp}$ [13]: $130-132{ }^{\circ} \mathrm{C} ;{ }^{31} \mathrm{P}\left(\mathrm{CDCl}_{3}\right) \delta 21.8$; $\delta[13] 21.6 ;[\mathrm{M}+\mathrm{H}]^{+}$found $=332.1050, \mathrm{C}_{17} \mathrm{H}_{19} \mathrm{NO}_{4}$ P requires 332.1046.

\subsubsection{Diisopropyl (2-Benzyl-3-oxo-2,3-dihydro-1H-isoindol-1-yl)phosphonate (9c)}

Yield: $83 \%(0.32 \mathrm{~g})$, colourless oil; ${ }^{31} \mathrm{P}\left(\mathrm{CDCl}_{3}\right) \delta 16.6 ;{ }^{1} \mathrm{H} \mathrm{NMR}\left(\mathrm{CDCl}_{3}\right) \delta 0.85\left(\mathrm{~d}, J_{\mathrm{HH}}=6.2,3 \mathrm{H}\right.$, $\left.\mathrm{CH}_{3} \mathrm{CH}^{\mathrm{I}}\right), 1.16\left(\mathrm{~d}, J_{\mathrm{HH}}=6.2,3 \mathrm{H}, \mathrm{CH}_{3} \mathrm{CH}^{\mathrm{II}}\right), 1.19\left(\mathrm{~d}, J_{\mathrm{HH}}=6.1,3 \mathrm{H}, \mathrm{CH}_{3} \mathrm{CH}^{\mathrm{III}}\right), 1.23\left(\mathrm{~d}, J_{\mathrm{HH}}=6.2,3 \mathrm{H}\right.$, $\left.\mathrm{CH}_{3} \mathrm{CH}^{\mathrm{IV}}\right), 4.28-4.44\left(\mathrm{~m}, 1 \mathrm{H}, \mathrm{CHO}^{\mathrm{I}}\right), 4.50-4.74\left[4.56\left(\mathrm{~d}, J_{\mathrm{HP}}=15.0, \mathrm{CH}_{\mathrm{A}}, \mathrm{CH}_{2} \mathrm{~N}\right), 4.57\left(\mathrm{~d}, J_{\mathrm{HP}}=12.5, \mathrm{C}_{1} \mathrm{H}\right)\right.$ overlapped by the multiplet of $\mathrm{CHO}^{\mathrm{II}}$ total int. $\left.3 \mathrm{H}\right], 5.48\left(\mathrm{~d}, J_{\mathrm{HP}}=14.9,1 \mathrm{H}, \mathrm{CH}_{\mathrm{B}}, \mathrm{CH}_{2} \mathrm{~N}\right), 7.12-7.29(\mathrm{~m}, 5 \mathrm{H}$, $\left.\mathrm{C}_{2}{ }^{\prime}, \mathrm{H}\right), 7.39-7.55\left(\mathrm{~m}, 2 \mathrm{H}, \mathrm{C}_{5} \mathrm{H}, \mathrm{C}_{6} \mathrm{H}\right), 7.64\left(\mathrm{~d}, \mathrm{~J}_{\mathrm{HH}}=7.1,1 \mathrm{H}, \mathrm{C}_{7} \mathrm{H}\right), 7.84\left(\mathrm{~d}, \mathrm{~J}_{\mathrm{HH}}=6.8,1 \mathrm{H}, \mathrm{C}_{4} \mathrm{H}\right)$; ${ }^{13} \mathrm{C} \mathrm{NMR}\left(\mathrm{CDCl}_{3}\right) \delta 23.3\left(\mathrm{~d},{ }^{3} J_{\mathrm{CP}}=5.4, \mathrm{CH}_{3} \mathrm{CH}^{\mathrm{I}}\right), 23.9\left(\mathrm{~d},{ }^{3} J_{\mathrm{CP}}=5.1, \mathrm{CH}_{3} \mathrm{CH}^{\mathrm{II}}\right), 24.06\left(\mathrm{~d},{ }^{3} J_{\mathrm{CP}}=5.8\right.$, $\left.\mathrm{CH}_{3} \mathrm{CH}^{\mathrm{III}}\right), 24.11\left(\mathrm{~d},{ }^{3} J_{\mathrm{CP}}=5.4, \mathrm{CH}_{3} \mathrm{CH}^{\mathrm{IV}}\right), 44.9\left(\mathrm{CH}_{2} \mathrm{~N}\right), 56.9\left(\mathrm{~d},{ }^{1} J_{\mathrm{CP}}=156.5, \mathrm{C}_{1}\right), 72.3\left(\mathrm{~d},{ }^{2} J_{\mathrm{CP}}=7.4\right.$, $\left.\mathrm{CHO}^{\mathrm{I}}\right), 72.6\left(\mathrm{~d},{ }^{2} J_{\mathrm{CP}}=7.2, \mathrm{CHO}^{\mathrm{II}}\right), 123.9\left(\mathrm{~d}, J_{\mathrm{CP}}=1.6, \mathrm{C}_{4}\right), 124.5\left(\mathrm{~d},{ }^{3} J_{\mathrm{CP}}=2.5, \mathrm{C}_{7}\right), 127.6\left(\mathrm{C}_{4^{\prime}}\right), 128.4$ $\left(\mathrm{C}_{3^{\prime}}\right), 128.68\left(\mathrm{C}_{2^{\prime}}\right), 128.70\left(\mathrm{~d}, J_{\mathrm{CP}}=2.6, \mathrm{C}_{5}\right), 131.6\left(\mathrm{~d}, J_{\mathrm{CP}}=2.6, \mathrm{C}_{6}\right), 132.2\left(\mathrm{~d},{ }^{3} J_{\mathrm{CP}}=4.3, \mathrm{C}_{3 \mathrm{a}}\right), 136.9$ $\left(\mathrm{C}_{1^{\prime}}\right), 139.0\left(\mathrm{~d},{ }^{2} J_{\mathrm{CP}}=5.8, \mathrm{C}_{7 \mathrm{a}}\right), 168.9\left(\mathrm{~d},{ }^{3} J_{\mathrm{CP}}=3.7, \mathrm{C}_{3}\right) ;[\mathrm{M}+\mathrm{H}]^{+}$found $=388.1667, \mathrm{C}_{21} \mathrm{H}_{27} \mathrm{NO}_{4} \mathrm{P}$ requires 388.1672 .

\subsubsection{Dibutyl (2-Benzyl-3-oxo-2,3-dihydro-1H-isoindol-1-yl)phosphonate (9d)}

Yield: $85 \%$ (0.35 g), colourless oil; ${ }^{31} \mathrm{P}\left(\mathrm{CDCl}_{3}\right) \delta 18.3 ;{ }^{1} \mathrm{H} \mathrm{NMR}\left(\mathrm{CDCl}_{3}\right) \delta 0.83\left(\mathrm{t}, J_{\mathrm{HH}}=7.4,3 \mathrm{H}\right.$, $\left.\left(\mathrm{CH}_{3} \mathrm{CH}_{2}\right)_{3} \mathrm{O}^{\mathrm{I}}\right), 0.89\left(\mathrm{t}, \mathrm{J}_{\mathrm{HH}}=7.4,3 \mathrm{H}, \mathrm{CH}_{3}\left(\mathrm{CH}_{2}\right)_{3} \mathrm{O}^{\mathrm{II}}\right), 1.15-1.36\left(\mathrm{~m}, 4 \mathrm{H}, \mathrm{CH}_{2}\left(\mathrm{CH}_{2}\right)_{2} \mathrm{O}\right), 1.36-1.50(\mathrm{~m}, 2 \mathrm{H}$, $\left.\mathrm{CH}_{2} \mathrm{CH}_{2} \mathrm{O}^{\mathrm{I}}\right), 1.52-1.64\left(\mathrm{~m}, 2 \mathrm{H}, \mathrm{CH}_{2} \mathrm{CH}_{2} \mathrm{O}^{\mathrm{II}}\right), 3.67-3.79\left(\mathrm{~m}, 1 \mathrm{H}, \mathrm{CH}_{\mathrm{A}}, \mathrm{CH}_{2} \mathrm{O}^{\mathrm{I}}\right), 3.84-3.79\left(\mathrm{~m}, 1 \mathrm{H}, \mathrm{CH}_{\mathrm{B}}\right.$, $\left.\mathrm{CH}_{2} \mathrm{O}^{\mathrm{I}}\right), 3.98-4.10\left(\mathrm{~m}, 2 \mathrm{H}, \mathrm{CH}_{2} \mathrm{O}^{\mathrm{II}}\right), 4.6\left(\mathrm{~d}, \mathrm{~J}_{\mathrm{HP}}=14.9,1 \mathrm{H}, \mathrm{CH}_{\mathrm{A}}, \mathrm{CH}_{2} \mathrm{~N}\right), 4.68\left(\mathrm{~d}, J_{\mathrm{HP}}=13.3,1 \mathrm{H}\right.$, $\left.\mathrm{C}_{1} \mathrm{H}\right), 5.57\left(\mathrm{~d}, J_{\mathrm{HP}}=14.8,1 \mathrm{H}, \mathrm{CH}_{\mathrm{B}}, \mathrm{CH}_{2} \mathrm{~N}\right), 7.21-7.34\left(\mathrm{~m}, 5 \mathrm{H}, \mathrm{C}_{2}-4^{\prime} \mathrm{H}\right), 7.47-7.60\left(\mathrm{~m}, 2 \mathrm{H}, \mathrm{C}_{5} \mathrm{H}, \mathrm{C}_{6} \mathrm{H}\right)$, $7.70\left(\mathrm{~d}, J_{\mathrm{HH}}=7.0,1 \mathrm{H}, \mathrm{C}_{7} \mathrm{H}\right), 7.92\left(\mathrm{~d}, J_{\mathrm{HH}}=6.7,1 \mathrm{H}, \mathrm{C}_{4} \mathrm{H}\right) ;{ }^{13} \mathrm{C} \mathrm{NMR}\left(\mathrm{CDCl}_{3}\right) 13.4\left(\mathrm{CH}_{3}\left(\mathrm{CH}_{2}\right)_{3} \mathrm{O}^{\mathrm{I}}\right)$, $13.5\left(\mathrm{CH}_{3}\left(\mathrm{CH}_{2}\right)_{3} \mathrm{O}^{\mathrm{II}}\right), 18.5\left(\mathrm{CH}_{2}\left(\mathrm{CH}_{2}\right)_{2} \mathrm{O}^{\mathrm{I}}\right), 18.6\left(\mathrm{CH}_{2}\left(\mathrm{CH}_{2}\right)_{2} \mathrm{O}^{\mathrm{II}}\right), 32.3\left(\mathrm{~d},{ }^{3} \mathrm{~J}_{\mathrm{CP}}=5.6, \mathrm{CH}_{2} \mathrm{CH}_{2} \mathrm{O}^{\mathrm{I}}\right)$, $32.5\left(\mathrm{~d},{ }^{3} J_{\mathrm{CP}}=5.6, \mathrm{CH}_{2} \mathrm{CH}_{2} \mathrm{O}^{\mathrm{II}}\right), 45.0\left(\mathrm{CH}_{2} \mathrm{~N}\right), 56.4\left(\mathrm{~d},{ }^{1} J_{\mathrm{CP}}=155.5, \mathrm{C}_{1}\right), 66.9\left(\mathrm{~d},{ }^{2} J_{\mathrm{CP}}=7.4, \mathrm{CH}_{2} \mathrm{O}^{\mathrm{I}}\right)$, $67.1\left(\mathrm{~d},{ }^{2} \mathrm{~J}_{\mathrm{CP}}=7.3, \mathrm{CH}_{2} \mathrm{O}^{\mathrm{II}}\right), 124.0\left(\mathrm{~d}, J_{\mathrm{CP}}=1.7, \mathrm{C}_{4}\right), 124.5\left(\mathrm{~d},{ }^{3} J_{\mathrm{CP}}=2.6, \mathrm{C}_{7}\right), 127.6\left(\mathrm{C}_{4^{\prime}}\right), 128.3$ $\left(C_{3^{\prime}}\right), 128.7\left(C_{2^{\prime}}\right), 128.8\left(\mathrm{~d}, J_{\mathrm{CP}}=2.4, \mathrm{C}_{5}\right), 131.6\left(\mathrm{~d}, J_{\mathrm{CP}}=2.5, \mathrm{C}_{6}\right), 132.0\left(\mathrm{~d},{ }^{3} J_{\mathrm{CP}}=4.3, \mathrm{C}_{3 \mathrm{a}}\right), 136.8$ 
$\left(\mathrm{C}_{1^{\prime}}\right), 138.8\left(\mathrm{~d},{ }^{2} J_{\mathrm{CP}}=5.7, \mathrm{C}_{7 \mathrm{a}}\right), 168.8\left(\mathrm{~d},{ }^{3} J_{\mathrm{CP}}=3.6, \mathrm{C}_{3}\right) ;[\mathrm{M}+\mathrm{H}]^{+}$found $=416.1979, \mathrm{C}_{23} \mathrm{H}_{31} \mathrm{NO}_{4} \mathrm{P}$ requires 416.1985 .

\subsubsection{Dibenzyl (2-Benzyl-3-oxo-2,3-dihydro-1H-isoindol-1-yl)phosphonate (9e)}

Yield: $84 \%(0.33 \mathrm{~g})$, colourless oil; ${ }^{31} \mathrm{P}\left(\mathrm{CDCl}_{3}\right) \delta 19.2 ;{ }^{1} \mathrm{H}$ NMR $\left(\mathrm{CDCl}_{3}\right) \delta 4.49\left(\mathrm{~d}, J_{\mathrm{HP}}=14.9,1 \mathrm{H}\right.$, $\left.\mathrm{CH}_{\mathrm{A}}, \mathrm{CH}_{2} \mathrm{~N}\right), 4.61-4.74$ [4.65 $\left(\mathrm{d}, J_{\mathrm{HP}}=13.0, \mathrm{C}_{1} \mathrm{H}\right)$ overlapped by the multiplet of $\mathrm{CH}_{\mathrm{A}}, \mathrm{CH}_{2} \mathrm{O}^{\mathrm{I}}$ total int. $2 \mathrm{H}], 4.79-4.86\left(\mathrm{~m}, 1 \mathrm{H}, \mathrm{CH}_{\mathrm{B}}, \mathrm{CH}_{2} \mathrm{O}^{\mathrm{I}}\right), 4.92-5.02\left(\mathrm{~m}, 2 \mathrm{H}, \mathrm{CH}_{2} \mathrm{O}^{\mathrm{II}}\right), 5.48\left(\mathrm{~d}, J_{\mathrm{HP}}=14.9,1 \mathrm{H}, \mathrm{CH}_{\mathrm{B}}\right.$, $\left.\mathrm{CH}_{2} \mathrm{~N}\right), 7.10-7.18(\mathrm{~m}, 4 \mathrm{H}, \mathrm{ArH}), 7.18-7.25(\mathrm{~m}, 5 \mathrm{H}, \mathrm{ArH}), 7.27-7.31(\mathrm{~m}, 3 \mathrm{H}, \mathrm{ArH}), 7.31-7.39(\mathrm{~m}, 3 \mathrm{H}$, $\operatorname{ArH})$, 7.45-7.55 (m, 2H, $\left.\mathrm{C}_{5} \mathrm{H}, \mathrm{C}_{6} \mathrm{H}\right), 7.64\left(\mathrm{~d}, \mathrm{~J}_{\mathrm{HH}}=6.9,1 \mathrm{H}, \mathrm{C}_{7} \mathrm{H}\right), 7.87\left(\mathrm{~d}, \mathrm{~J}_{\mathrm{HH}}=5.8,1 \mathrm{H}, \mathrm{C}_{4} \mathrm{H}\right) ;{ }^{13} \mathrm{C}$ $\operatorname{NMR}\left(\mathrm{CDCl}_{3}\right) 45.0\left(\mathrm{CH}_{2} \mathrm{~N}\right), 56.6\left(\mathrm{~d},{ }^{1} J_{\mathrm{CP}}=155.5, \mathrm{C}_{1}\right), 68.6\left(\mathrm{~d},{ }^{2} J_{\mathrm{CP}}=7.3, \mathrm{CH}_{2} \mathrm{O}^{\mathrm{I}}\right), 68.9\left(\mathrm{~d},{ }^{2} J_{\mathrm{CP}}=6.9\right.$, $\left.\mathrm{CH}_{2} \mathrm{O}^{\mathrm{II}}\right), 124.1\left(\mathrm{~d}, J_{\mathrm{CP}}=1.7, \mathrm{C}_{4}\right), 124.4\left(\mathrm{~d},{ }^{3} J_{\mathrm{CP}}=2.6, \mathrm{C}_{7}\right), 127.6\left(\mathrm{C}_{4^{\prime}}\right), 128.1\left(\mathrm{C}_{3^{\prime \prime}}{ }^{\prime} \mathrm{I}\right), 128.3\left(\mathrm{C}_{3^{\prime \prime}}{ }^{\prime \prime}\right), 128.4$ $\left(\mathrm{C}_{3^{\prime}}\right), 128.65\left(\mathrm{C}_{2^{\prime \prime}} \mathrm{I}\right), 128.66\left(\mathrm{C}_{2^{\prime}}\right), 128.67\left(\mathrm{C}_{2^{\prime \prime}} \mathrm{II}\right), 128.71\left(\mathrm{C}_{4^{\prime \prime}} \mathrm{I}\right), 128.8\left(\mathrm{C}_{4^{\prime \prime}} \mathrm{II}\right), 128.9\left(\mathrm{~d}, J_{\mathrm{CP}}=2.3, \mathrm{C}_{5}\right)$, $131.7\left(\mathrm{~d}, J_{\mathrm{CP}}=2.6, \mathrm{C}_{6}\right), 132.0\left(\mathrm{~d},{ }^{3} J_{\mathrm{CP}}=4.1, \mathrm{C}_{3 \mathrm{a}}\right), 135.36\left(\mathrm{~d},{ }^{3} J_{\mathrm{CP}}=5.1, \mathrm{C}_{1^{\prime \prime}}{ }^{\prime \prime}\right), 135.40\left(\mathrm{~d},{ }^{3} J_{\mathrm{CP}}=5.5, \mathrm{C}_{1^{\prime}},{ }^{\prime \prime}\right)$, $136.7\left(\mathrm{C}_{1^{\prime}}\right), 138.4\left(\mathrm{~d},{ }^{2} J_{\mathrm{CP}}=5.8, \mathrm{C}_{7 \mathrm{a}}\right), 168.7\left(\mathrm{~d},{ }^{3} J_{\mathrm{CP}}=3.7, \mathrm{C}_{3}\right) ;[\mathrm{M}+\mathrm{H}]^{+}$found $=484.1679, \mathrm{C}_{29} \mathrm{H}_{27} \mathrm{NO}_{4} \mathrm{P}$ requires 484.1672 .

\subsubsection{Ethyl (2-Butyl-3-oxo-2,3-dihydro-1H-isoindol-1-yl)(phenyl)phosphinate (10)}

Yield: $78 \%(0.28 \mathrm{~g})$, colourless oil; $[\mathrm{M}+\mathrm{H}]^{+}$found $=358.1558, \mathrm{C}_{20} \mathrm{H}_{25} \mathrm{NO}_{3} \mathrm{P}$ requires 358.1566; Product $\mathbf{1 0}$ formed as a mixture of two isomers ( $\mathbf{A}$ and $\mathbf{B}$ ) in a proportion of 47:53 based on ${ }^{31} \mathrm{P}$ NMR. The two series of signals could be separated in the NMR spectra: Isomer A: ${ }^{31} \mathrm{P}\left(\mathrm{CDCl}_{3}\right) \delta 35.4 ;{ }^{1} \mathrm{H}$ $\operatorname{NMR}\left(\mathrm{CDCl}_{3}\right) \delta 0.94\left(\mathrm{t}, J_{\mathrm{HH}}=7.4,3 \mathrm{H}, \mathrm{CH}_{3}\left(\mathrm{CH}_{2}\right)_{3} \mathrm{~N}\right), 1.23-1.38\left(\mathrm{~m}, 2 \mathrm{H}, \mathrm{CH}_{2}\left(\mathrm{CH}_{2}\right)_{2} \mathrm{~N}\right), 1.43\left(\mathrm{t}, J_{\mathrm{HH}}=7.0\right.$, $\left.3 \mathrm{H}, \mathrm{CH}_{3} \mathrm{CH}_{2} \mathrm{O}\right), 1.54-1.80\left(\mathrm{~m}, 2 \mathrm{H}, \mathrm{CH}_{2} \mathrm{CH}_{2} \mathrm{~N}\right), 3.66-3.78\left(\mathrm{~m}, 1 \mathrm{H}, \mathrm{CH}_{\mathrm{A}}, \mathrm{CH}_{2} \mathrm{~N}\right), 4.19-4.39\left(\mathrm{~m}, 3 \mathrm{H}, \mathrm{CH}_{\mathrm{B}}\right.$, $\left.\mathrm{CH}_{2} \mathrm{~N}, \mathrm{CH}_{2} \mathrm{O}\right), 5.05\left(\mathrm{~d}, J_{\mathrm{HP}}=16.7,1 \mathrm{H}, \mathrm{C}_{1} \mathrm{H}\right), 7.12-7.67\left(\mathrm{~m}, 8 \mathrm{H}, \mathrm{ArH}, \mathrm{C}_{5-7} \mathrm{H}\right), 7.75\left(\mathrm{~d}, J_{\mathrm{HH}}=7.8,1 \mathrm{H}\right.$, $\left.\mathrm{C}_{4} \mathrm{H}\right) ;{ }^{13} \mathrm{C} \mathrm{NMR}\left(\mathrm{CDCl}_{3}\right) \delta 13.7\left(\mathrm{CH}_{3}\left(\mathrm{CH}_{2}\right)_{3} \mathrm{~N}\right), 16.50\left(\mathrm{~d},{ }^{3} J_{\mathrm{CP}}=6.1, \mathrm{CH}_{3} \mathrm{CH}_{2} \mathrm{O}\right), 20.0\left(\mathrm{CH}_{2}\left(\mathrm{CH}_{2}\right)_{2} \mathrm{~N}\right)$, $30.02\left(\mathrm{CH}_{2} \mathrm{CH}_{2} \mathrm{~N}\right), 41.5\left(\mathrm{CH}_{2} \mathrm{~N}\right), 60.1\left(\mathrm{~d},{ }^{1} J_{\mathrm{CP}}=107.9, \mathrm{C}_{1}\right), 61.9\left(\mathrm{~d},{ }^{2} J_{\mathrm{CP}}=6.7, \mathrm{CH}_{2} \mathrm{O}\right), 123.4\left(\mathrm{~d}, J_{\mathrm{CP}}=1.6\right.$, $\left.\mathrm{C}_{4}\right), 123.7\left(\mathrm{~d},{ }^{3} J_{\mathrm{CP}}=1.7, \mathrm{C}_{7}\right), 124.45\left(\mathrm{~d},{ }^{3} J_{\mathrm{CP}}=23.1, \mathrm{C}_{3^{\prime}}\right), 125.5\left(\mathrm{~d},{ }^{1} J_{\mathrm{CP}}=127.3, \mathrm{C}_{1^{\prime}}\right), 128.21\left(\mathrm{~d},{ }^{2} J_{\mathrm{CP}}=12.7\right.$, $\left.\mathrm{C}_{2^{\prime}}\right), 128.67\left(\mathrm{~d}, J_{\mathrm{CP}}=2.0, \mathrm{C}_{5}\right), 131.1\left(\mathrm{~d}, J_{\mathrm{CP}}=2.3, \mathrm{C}_{6}\right), 132.1\left(\mathrm{~d}, J_{\mathrm{CP}}=9.4, \mathrm{C}_{4^{\prime}}\right), 133.04\left(\mathrm{~d},{ }^{3} J_{\mathrm{CP}}=3.0\right.$, $\left.\mathrm{C}_{3 \mathrm{a}}\right), 138.7\left(\mathrm{~d},{ }^{2} J_{\mathrm{CP}}=4.1, \mathrm{C}_{7 \mathrm{a}}\right), 168.3\left(\mathrm{~d},{ }^{3} J_{\mathrm{CP}}=2.6, \mathrm{C}_{3}\right)$; Isomer $\mathrm{B}:{ }^{31} \mathrm{P}\left(\mathrm{CDCl}_{3}\right) \delta 39.2 ;{ }^{1} \mathrm{H} \mathrm{NMR}$ $\left(\mathrm{CDCl}_{3}\right) \delta 0.95\left(\mathrm{t}, J_{\mathrm{HH}}=7.4,3 \mathrm{H}, \mathrm{CH}_{3}\left(\mathrm{CH}_{2}\right)_{3} \mathrm{~N}\right), 1.23-1.38\left(\mathrm{~m}, 2 \mathrm{H}, \mathrm{CH}_{2}\left(\mathrm{CH}_{2}\right)_{2} \mathrm{~N}\right), 1.45\left(\mathrm{t}, J_{\mathrm{HH}}=7.0,3 \mathrm{H}\right.$, $\left.\mathrm{CH}_{3} \mathrm{CH}_{2} \mathrm{O}\right), 1.54-1.80\left(\mathrm{~m}, 2 \mathrm{H}, \mathrm{CH}_{2} \mathrm{CH}_{2} \mathrm{~N}\right), 3.51-3.66\left(\mathrm{~m}, 1 \mathrm{H}, \mathrm{CH}_{\mathrm{A}}, \mathrm{CH}_{2} \mathrm{~N}\right), 3.98-4.19\left(\mathrm{~m}, 3 \mathrm{H}, \mathrm{CH}_{\mathrm{B}}\right.$, $\left.\mathrm{CH}_{2} \mathrm{~N}, \mathrm{CH}_{2} \mathrm{O}\right), 5.05\left(\mathrm{~d}, J_{\mathrm{HP}}=16.7,1 \mathrm{H}, \mathrm{C}_{1} \mathrm{H}\right), 7.12-7.67\left(\mathrm{~m}, 8 \mathrm{H}, \mathrm{ArH}, \mathrm{C}_{5-7} \mathrm{H}\right), 7.86\left(\mathrm{~d}, J_{\mathrm{HH}}=7.7,1 \mathrm{H}\right.$, $\left.\mathrm{C}_{4} \mathrm{H}\right) ;{ }^{13} \mathrm{C} \mathrm{NMR}\left(\mathrm{CDCl}_{3}\right) \delta 13.7\left(\mathrm{CH}_{3}\left(\mathrm{CH}_{2}\right)_{3} \mathrm{~N}\right), 16.54\left(\mathrm{~d},{ }^{3} J_{\mathrm{CP}}=6.1, \mathrm{CH}_{3} \mathrm{CH}_{2} \mathrm{O}\right), 20.0\left(\mathrm{CH}_{2}\left(\mathrm{CH}_{2}\right)_{2} \mathrm{~N}\right)$, $29.98\left(\mathrm{CH}_{2} \mathrm{CH}_{2} \mathrm{~N}\right), 41.4\left(\mathrm{CH}_{2} \mathrm{~N}\right), 59.4\left(\mathrm{~d},{ }^{1} J_{\mathrm{CP}}=104.5, \mathrm{C}_{1}\right), 62.2\left(\mathrm{~d},{ }^{2} J_{\mathrm{CP}}=7.1, \mathrm{CH}_{2} \mathrm{O}\right), 123.4\left(\mathrm{~d}, J_{\mathrm{CP}}=1.6\right.$, $\left.\mathrm{C}_{4}\right), 123.7\left(\mathrm{~d},{ }^{3} J_{\mathrm{CP}}=1.7, \mathrm{C}_{7}\right), 124.42\left(\mathrm{~d},{ }^{3} J_{\mathrm{CP}}=22.8, \mathrm{C}_{3^{\prime}}\right), 125.1\left(\mathrm{~d},{ }^{1} J_{\mathrm{CP}}=128.4, \mathrm{C}_{1^{\prime}}\right), 128.25\left(\mathrm{~d},{ }^{2} J_{\mathrm{CP}}=12.8\right.$, $\left.\mathrm{C}_{2^{\prime}}\right), 128.60\left(\mathrm{~d}, J_{\mathrm{CP}}=2.5, \mathrm{C}_{5}\right), 131.3\left(\mathrm{~d}, J_{\mathrm{CP}}=2.6, \mathrm{C}_{6}\right), 131.9\left(\mathrm{~d}, J_{\mathrm{CP}}=9.3, \mathrm{C}_{4^{\prime}}\right), 132.98\left(\mathrm{~d},{ }^{3} J_{\mathrm{CP}}=3.3, \mathrm{C}_{3 \mathrm{a}}\right)$, $138.1\left(\mathrm{~d},{ }^{2} J_{\mathrm{CP}}=1.8, \mathrm{C}_{7 \mathrm{a}}\right), 168.7\left(\mathrm{~d},{ }^{3} J_{\mathrm{CP}}=2.1, \mathrm{C}_{3}\right)$.

\subsection{Procedure for the Real Time Monitoring of the Condensation of 2-Formylbenzoic Acid, Butylamine and} Diethyl Phosphite by In Situ FT-IR Spectroscopy

To $30 \mathrm{~mL}$ of ethanol, the starting materials were mixed at room temperature $\left(25^{\circ} \mathrm{C}\right)$ in ten-minute intervals in the following order: $30 \mathrm{mmol}(4.5 \mathrm{~g})$ of 2-formylbenzoic acid, $30 \mathrm{mmol}(3.9 \mathrm{~mL})$ of diethyl phosphite and $36 \mathrm{mmol}(3.6 \mathrm{~mL})$ of butylamine. After the additions, the mixture was heated gradually from $25{ }^{\circ} \mathrm{C}$ to $60{ }^{\circ} \mathrm{C}$ in an oil bath. Then the temperature was held for an additional $2.5 \mathrm{~h}$.

\subsection{Procedure for the Continuous Flow Synthesis of Diethyl} (2-butyl-3-oxo-2,3-dihydro-1H-isoindol-1-yl)phosphonate (7a)

Before each experiment, two solutions (A and B) were prepared. For solution A, $41.8 \mathrm{mmol}$ of 2-formylbenzoic acid was dissolved in ethanol in a $50 \mathrm{~cm}^{3}$ volumetric flask, resulting in the aldehyde concentration of $0.84 \mathrm{M}$. For solution B, amines $(50.2 \mathrm{mmol}$ of butylamine $(5.0 \mathrm{~mL})$ or $62.7 \mathrm{mmol}$ of butylamine $(6.2 \mathrm{~mL}), 62.7 \mathrm{mmol}$ of cyclohexylamine $(7.2 \mathrm{~mL})$ or $62.7 \mathrm{mmol}$ of 
benzylamine $(6.8 \mathrm{~mL}))$ and $41.8 \mathrm{mmol}$ of diethyl phosphite $(5.4 \mathrm{~mL})$ or $62.7 \mathrm{mmol}$ of diethyl phosphite $(8.1 \mathrm{~mL})$ were dissolved in ethanol in a $50 \mathrm{~cm}^{3}$ volumetric flask, resulting in the amine concentration of $1.01 \mathrm{M}$ or $1.26 \mathrm{M}$ and the dialkyl phosphite concentration of $0.84 \mathrm{M}$ or $1.26 \mathrm{M}$, respectively. The two solutions (A and B) were pumped separately. The system was flushed with $15 \mathrm{~mL}$ of solution $\mathrm{A}$ and $15 \mathrm{~mL}$ of solution $\mathrm{B}$ with a summa flow rate of $10 \mathrm{~mL} / \mathrm{min}(\mathrm{A}: \mathrm{B}=1: 1)$ at $25{ }^{\circ} \mathrm{C}$ and 17 bar. Next, under the same pressure, the flow rate was set to the desired value (can be seen in Table 2) and the vessel was irradiated with a power of 10-15 W for 2-3 min, until the temperature reached the desired value, then the power was controlled automatically by the software of the MW reactor. The operation was regarded steady state on the basis of the results of the GC measurements. The desired diethyl (2-butyl-3-oxo-2,3-dihydro- $1 H$-isoindol-1-yl)phosphonate (7a), diethyl (2-cyclohexyl-3-oxo-2,3-dihydro-1H-isoindol-1-yl)phosphonate (8a) or diethyl (2-benzyl-3-oxo2,3-dihydro- $1 H$-isoindol-1-yl)phosphonate (9a) were obtained by column chromatography using silica gel as the absorbent and dichloromethane:methanol (97:3) as the eluent. Yields were calculated on the basis of the weights obtained after separation and evaporation taking into consideration the quantity of the limiting starting materials fed in during a given time. After a $3 \mathrm{~h}$ flow operation in steady state, $6.9 \mathrm{~g}$ of diethyl (2-butyl-3-oxo-2,3-dihydro- $1 H$-isoindol-1-yl)phosphonate (7a), $4.1 \mathrm{~g}$ of diethyl (2-cyclohexyl-3-oxo-2,3-dihydro- $1 \mathrm{H}$-isoindol-1-yl)phosphonate (8a) or $5.3 \mathrm{~g}$ of diethyl (2-benzyl-3-oxo-2,3-dihydro-1H-isoindol-1-yl)phosphonate (9a) were obtained.

\section{Conclusions}

In conclusion, we have developed a facile, efficient, catalyst-free method for the batch and continuous flow preparation of isoindolin-1-one-3-phosphonates containing alkyl substituents on the nitrogen atom. The novel batch approach enabled the solvent-free synthesis of the target compounds in high yields $(70-94 \%)$ at low temperature $\left(60^{\circ} \mathrm{C}\right)$ under short reaction times $(10-30 \mathrm{~min})$. By the in situ FT-IR study on the condensation of 2-formylbenzoic acid, butylamine and diethyl phosphite, the reaction was followed in "real time" and the time-dependent concentration profiles of the reaction components were determined. The continuous flow MW-assisted method developed for the condensation of 2-formylbenzoic acid, aliphatic primary amines and diethyl phosphite are suitable for the synthesis of the corresponding isoindolin-1-one-3-phosphonates in a "few g" scale. Altogether 16 derivatives were isolated and characterized. Except two, all of them are new compounds.

Supplementary Materials: Supplementary materials are available online.

Author Contributions: E.B. and Á.T. planned the experiments, N.T. and. B.R. carried out the experiments, I.C. contributed to the Fourier transform FT-IR reaction monitoring, P.T.S. performed the high resolution mass spectrometric measurements, E.B., Á.T. and N.T. wrote the paper. All authors have read and agreed to the published version of the manuscript.

Funding: The above project was supported by the Hungarian Research Development and Innovation Office (FK123961), by the National Research, Development and Innovation Fund of Hungary in the frame of FIEK_16-1-2016-0007 (Higher Education and Industrial Cooperation Center) project. N.T. was supported by the Servier-Beregi PhD Research Fellowship. E.B. was supported by the János Bolyai Research Scholarship of the Hungarian Academy of Sciences (BO/00278/17/7).

Conflicts of Interest: The authors declare no conflict of interest.

\section{References}

1. Bhatia, R.K. Isoindole derivatives: Propitious anticancer structural motifs. Curr. Top. Med. Chem. 2017, 17, 189-207. [CrossRef] [PubMed]

2. Palillero-Cisneros, A.; Bedolla-Medrano, M.; Ordóñez, M. Efficient $\mathrm{PhB}(\mathrm{OH})_{2}$-catalyzed one-pot synthesis of 3-substituted isoindolin-1-ones and isobenzofuran-1(3H)-ones under solvent free conditions. Tetrahedron 2018, 74, 4174-4181. [CrossRef] 
3. Ordóñez, M.; Palillero-Cisneros, A.; Labastida-Galván, V.; Terán-Vázquez, J.L. Practical synthesis of 3-(2-arylethylidene)isoindolin-1-ones (analogues of AKS-182) and 3-(2-arylethylidene)isobenzofuran1(3H)-ones. Tetrahedron 2020, 76, 130838. [CrossRef]

4. Wang, J.; Teng, Q.; Lin, C.; Gao, F.; Liu, X.; Shen, L. Co(II)-Catalyzed desilylative annulation of benzamides and acrylamides with alkynylsilanes: Access to 3-methyleneisoindolin-1-one and 5-methylene-1H-pyrrol-2(5H)-one derivatives. Synthesis 2020, 52, 1969-1980. [CrossRef]

5. Gordon, C.P.; Byrne, N.; McCluskey, A. A facile, protic ionic liquid route to $N$-substituted 5-hydroxy-4-methyl-3-oxoisoindoline-1-carboxamides and $\mathrm{N}$-substituted 3-oxoisoindoline-4-carboxylic acids. Green Chem. 2010, 12, 1000-1006. [CrossRef]

6. Couture, A.; Moreau, A.; Deniau, E.; Grandclaudon, P. A convenient synthesis of 4-alkoxylated isoindolin-1-ones. Synthesis 2004, 10, 1664-1670. [CrossRef]

7. Bunce, R.A.; Muddala, N.P.; Nammalwar, B. Expeditous synthesis of ( \pm )-diethyl 2-alkyl- and 2-aryl-(3-oxoisoindolin-1-yl)phosphonates catalysed by OSU-6. RSC Adv. 2015, 5, 28389-28393. [CrossRef]

8. Macsari, I.; Besidski, Y.; Csjernyik, G.; Nilsson, L.I.; Sandberg, L.; Yngve, U.; Ahlin, K.; Bueters, T.; Eriksson, A.B.; Lund, P.-E.; et al. 3 Oxoisoindoline-1-carboxamides: Potent, state-dependent blockers of voltage-gated sodium channel Nav1.7 with efficacy in rat pain models. J. Med. Chem. 2012, 55, 6866-6880. [CrossRef]

9. Bjore, A.; Bostrom, J.; Davidsson, O.; Emtenas, H.; Gran, U.; Iliefski, T.; Kajanus, J.; Olsson, R.; Strandlund, G.; Sundell, J.; et al. New Compounds I/418. U.S. Patent 001,523,7A1, January 2008.

10. Kukhar, V.P.; Hudson, H.R. Aminophosphonic and Aminophosphinic Acids: Chemistry and Biological Activity; Wiley: Chichester, UK, 2000; Volume 14, ISBN 978-0-471-89149-9.

11. Phillips, W.G. Phosphinylphthalimidines and their use as plant growth regulants. U.S. Patent 4,164,406, August 1979.

12. Ordóñez, M.; Tibhe, G.D.; Zamudio-Medina, A.; Viveros-Ceballos, J.V. An easy approach for the synthesis of $\mathrm{N}$-substituted isoindolin-1-ones. Synthesis 2012, 44, 569-574. [CrossRef]

13. Ordóñez, M.; Reyes-González, M.A.; Zamudio-Medina, A. Practical and high stereoselective synthesis of 3-(arylmethylene)isoindolin-1-ones from 2-formylbenzoic acid. Tetrahedron Lett. 2012, 53, 5756-5758. [CrossRef]

14. Reyes-González, M.A.; Zamudio-Medina, A.; Ramírez-Marroquin, O.A.; Ordóñez, M. Synthesis of 3-alkylideneisoindolinones and isoindolones by a Horner-Wadsworth-Emmons reaction. Monatsch. Chem. 2014, 145, 1001-1007. [CrossRef]

15. Ordóñez, M.; Viveros-Ceballos, J.L.; Cativiela, C. One-pot three-component highly diastereoselective synthesis of isoindolin-1-one-3-phosphonates under solvent and catalyst free-conditions. Tetrahedron: Asymmetry 2011, 22, 1479-1484. [CrossRef]

16. Failla, S.; Finocchiaro, P. Unexpected course of dimethyl phosphite addition to the condensation products obtained from o-carboxybenzaldehyde and aliphatic amines. Phosphorus Sulfur Silicon Relat. Elem. 1995, 105, 195-203. [CrossRef]

17. Milen, M.; Dancsó, A.; Földesi, T.; Slégel, P.; Volk, B. Propylphosphonic anhydride (T3P) mediated one-pot three-component synthesis of racemic dialkyl (2-substituted3-oxo-2,3-dihydro$1 H$-isoindol-1-yl)phosphonates. Tetrahedron 2016, 72, 5091-5099. [CrossRef]

18. Keglevich, G.; Bálint, E. The Kabachnik-Fields reaction: Mechanism and synthetic use. Molecules 2012, 17, 12821-12835. [CrossRef]

19. Claeys, D.D.; Stevens, C.V.; Roman, B.I.; Van De Caveye, P.; Waroquier, M.; Van Speybroeck, V. Experimental and computational study of the ring opening of tricyclic oxanorbornenes to polyhydro isoindole phosphonates. Org. Biomol. Chem. 2010, 8, 3644-3654. [CrossRef]

20. Couture, A.; Deniau, E.; Woisel, P.; Grandclaudon, P. A short and efficient synthesis of 3-diphenylphosphoryland 3-diethoxyphosphoryl(aza)isoindolinones: Extension to the sulfonylated analogs. Synthesis 1997, 12, 1439-1445. [CrossRef]

21. Kachkovskyi, G.O.; Kolodiazhnyi, O.I. Synthesis of phosphonic acids possessing isoindolin-1-one moiety: Unexpected acid-catalyzed C-P-bond cleavage. Phosphorus Sulfur Silicon Relat. Elem. 2009, 184, 890-907. [CrossRef]

22. Kachkovskyi, G.O.; Kolodiazhnyi, O.I. Synthesis of the phosphonoanalogue of benzo[c]pyroglutamic acid. Phosphorus Sulfur Silicon Relat. Elem. 2010, 185, 2441-2448. [CrossRef] 
23. Kiss, N.Z.; Bálint, E.; Keglevich, G. Microwave-assisted syntheses in organic chemistry. In Milestones in Microwave Chemistry; Keglevich, G., Ed.; Springer: Basel, Switzerland, 2016; pp. 11-45, ISBN 978-3-319-30632-2.

24. Kappe, C.O.; Stadler, A.; Dallinger, D. Microwaves in organic and medicinal chemistry, 2nd ed.; Wiley: Weinheim, Germany, 2012; Volume 52, ISBN 978-3-527-33185-7.

25. Jiang, B.; Shi, F.; Tu, S.J. Microwave-assisted multicomponent reactions in the heterocyclic chemistry. Curr. Org. Chem. 2010, 14, 357-378. [CrossRef]

26. Bálint, E.; Keglevich, G. The Spread of the Application of the Microwave Technique in Organic Synthesis. In Milestones in Microwave Chemistry; Keglevich, G., Ed.; Springer: Basel, Switzerland, 2016; pp. 1-10, ISBN 978-3-319-30632-2.

27. de la Hoz, A.; Loupy, A. Microwaves in Organic Synthesis, 3rd ed.; Wiley: Weinheim, Germany, 2012; ISBN 978-3-527-65131-3.

28. Estela, L.; Pouxb, M.; Benamaraa, N.; Polaerta, I. Continuous flow-microwave reactor: Where are we? Chem. Eng. Process. 2016, 113, 56-64. [CrossRef]

29. Bálint, E.; Tajti, Á.; Ladányi-Pára, K.; Tóth, N.; Mátravölgyi, B.; Keglevich, G. Continuous flow synthesis of $\alpha$-aryl- $\alpha$-aminophosphonates. Pure Appl. Chem. 2019, 91, 67-76. [CrossRef]

30. Sterk, H. Untersuchungen zur Temperaturabhängigkeit charakteristischer IR-Absorptionen. Monatsch. Chem. 1968, 99, 1764-1769. [CrossRef]

31. Bowden, K.; Taylor, G.R. Ring-chain tautomerism. Part I. 2-Acyl- and 2-aroyl-benzoic acids. J. Chem. Soc. B 1971, 1390-1394. [CrossRef]

32. Bálint, E.; Tajti, Á.; Ádám, A.; Csontos, I.; Karaghiosoff, K.; Czugler, M.; Ábrányi-Balogh, P.; Keglevich, $\mathrm{G}$. The synthesis of $\alpha$-aryl- $\alpha$-aminophosphonates and $\alpha$-aryl- $\alpha$-aminophosphine oxides by the microwave-assisted Pudovik reaction. Beilstein J. Org. Chem. 2017, 13, 76-86. [CrossRef]

33. Tajti, Á.; Tóth, N.; Bálint, E.; Keglevich, G. Esterification of benzoic acid in a continuous flow microwave reactor. J. Flow Chem. 2018, 8, 11-19. [CrossRef]

34. Bálint, E.; Tajti, Á.; Tóth, N.; Keglevich, G. Continuous flow alcoholysis of dialkyl H-phosphonates with aliphatic alcohols. Molecules 2018, 23, 1618. [CrossRef]

Sample Availability: Samples of the compounds 7-10 are available from the authors.

(C) 2020 by the authors. Licensee MDPI, Basel, Switzerland. This article is an open access article distributed under the terms and conditions of the Creative Commons Attribution (CC BY) license (http://creativecommons.org/licenses/by/4.0/). 